\title{
OPTICAL SIGNATURES OF CIRCUMSTELLAR INTERACTION IN TYPE IIP SUPERNOVAE
}

\author{
Nikolai N. Chugai ${ }^{1,2}$, Roger A. Chevalier ${ }^{2}$, Victor P. Utrobin ${ }^{3}$
}

\begin{abstract}
We propose new diagnostics for circumstellar interaction in Type IIP supernovae (SNe IIP) by the detection of high velocity (HV) absorption features in $\mathrm{H} \alpha$ and HeI $10830 \AA$ lines during the photospheric stage. To demonstrate the method, we compute the ionization and excitation of $\mathrm{H}$ and $\mathrm{He}$ in supernova ejecta taking into account time-dependent effects and X-ray irradiation. We find that the interaction with a typical red supergiant wind should result in the enhanced excitation of the outer layers of unshocked ejecta and the emergence of corresponding $\mathrm{HV}$ absorption, i.e. a depression in the blue absorption wing of $\mathrm{H} \alpha$ and a pronounced absorption of HeI $10830 \AA$ at a radial velocity of about $-10^{4}$ $\mathrm{km} \mathrm{s}^{-1}$. We identify HV absorption in $\mathrm{H} \alpha$ and HeI $10830 \AA$ lines of SN $1999 \mathrm{em}$ and in $\mathrm{H} \alpha$ of SN 2004dj as being due to this effect. The derived mass loss rate is close to $10^{-6} M_{\odot} \mathrm{yr}^{-1}$ for both supernovae, assuming a wind velocity $10 \mathrm{~km}$ $\mathrm{s}^{-1}$. We argue that, in addition to the HV absorption formed in the unshocked ejecta, spectra of SN 2004dj and SN 1999em show a HV notch feature that is formed in the cool dense shell (CDS) modified by the Rayleigh-Taylor instability. The CDS results from both shock breakout and radiative cooling of gas that has passed through the reverse shock wave. The notch becomes dominant in the HV absorption during the late photospheric phase, $\gtrsim 60 \mathrm{~d}$. The wind density deduced from the velocity of the CDS is consistent with the wind density found from the $\mathrm{HV}$ absorption produced by unshocked ejecta.
\end{abstract}

Subject headings: stars: mass loss - supernovae: general - supernovae: individual (SN 1999em, SN 2004dj)

\footnotetext{
${ }^{1}$ Institute of Astronomy, RAS, Pyatnitskaya 48, 109017 Moscow, Russia; nchugai@inasan.ru

${ }^{2}$ Department of Astronomy, University of Virginia, P.O. Box 400325, Charlottesville, VA 22904; rac5x@virginia.edu

${ }^{3}$ Institute of Theoretical and Experimental Physics, 117218 Moscow, Russia; utrobin@itep.ru
} 


\section{INTRODUCTION}

The link between a certain Type IIP supernova (SN IIP) and its main-sequence progenitor is poorly known despite the widely accepted view that these supernovae arise from the initial mass range of $10-25 M_{\odot}$ (e.g., Heger et al. 2003). The primary reasons for the uncertainty in this field are the small number of presupernova (pre-SN) mass determinations and the uncertain amount of matter lost through winds, presumably dominated by the red supergiant (RSG) wind. While the former is becoming well-studied, the latter uncertainty is related to our poor knowledge of the complicated physics of mass loss and the unsatisfactory situation with the empirical measurements of mass loss from RSGs. Even for the most studied close massive RSG, Betelgeuse, the range of observational estimates is large: $2 \times 10^{-7}-1.5 \times 10^{-5} M_{\odot} \mathrm{yr}^{-1}$ (Jura \& Kleinmann 1990; van Loon et al. 2005).

The situation for SNe IIP has some promise because the dominant mass loss at the RSG stage may be observed by the detection of circumstellar (CS) interaction at X-ray and radio wavelengths. The Type IIP SN $1999 \mathrm{em}$ was detected in X-rays with Chandra (Pooley et al. 2002), leading to the mass loss estimate $\dot{M} \approx(1-2) \times 10^{-6} M_{\odot} \mathrm{yr}^{-1}$ (assuming a wind velocity of $10 \mathrm{~km} \mathrm{~s}^{-1}$ ). A recent study of available X-ray and radio data for SNe IIP produced a range of mass loss rates of pre-SN IIP $\dot{M} \sim(1-10) \times 10^{-6} M_{\odot} \mathrm{yr}^{-1}$ (Chevalier et al. 2006). The application of these rates to the full RSG stage $\left(\sim 10^{6} \mathrm{yr}\right)$ suggests the loss of $1-10 M_{\odot}$ for pre-SNe IIP. The wide range of estimates emphasizes the need for the individual determination of the wind density for each particular SN IIP under consideration. Unfortunately, this is not always possible since X-ray and radio observations of SNe IIP are often not available.

Here, we propose two new diagnostics for the wind density in SNe IIP that could help. Both rely on spectroscopic observations of $\mathrm{H} \alpha$ and He I $10830 \AA$ at the photospheric epoch. The first one is based on the fact that the interaction of SN ejecta with the wind results in the emission of X-rays from both forward and reverse shocks. The X-rays cause ionization and excitation of SN ejecta that may be revealed, e.g., through specific emission lines (Chevalier \& Fransson 1994). Unfortunately, in SNe IIP the wind density is low and emission lines caused by CS interaction are extremely weak and cannot be detected. We find, however, that the excitation of $\mathrm{H}$ and He produced by X-rays in SN IIP ejecta turns out to be sufficient to be detected as high velocity (HV) absorption features in $\mathrm{H} \alpha$ and He I 10830 $\AA$ lines against the bright SN IIP photosphere. This is the core of our proposed diagnostic for the wind in SNe IIP. The second proposed probe for CS interaction exploits the possibility that a cool dense shell (CDS) might form at the SN/CS interface because of radiative

cooling. The CDS excited by X-rays could become visible as narrow HV absorption in $\mathrm{H} \alpha$. The velocity of this absorption would provide a direct measure of the expansion velocity of 
the SN/CS interface, a valuable dynamical characteristic of the CS interaction.

The identification of the expected HV lines in observed spectra is complicated by the presence of weak metal lines (Dessart \& Hillier 2005). However, we argue that HV lines of $\mathrm{H} \alpha$ and $\mathrm{H} \beta$ have been observed in spectra of SNe IIP, as previously discussed by Leonard et al. (2002a) for SN 1999em.

The paper is organized as follows. We start with a description of the interaction model and CS interaction effects in the $\mathrm{H} \alpha$ and HeI $10830 \AA$ absorption lines formed in the unshocked ejecta during the photospheric epoch ( $\S 2$ 2). We then compare our CS interaction models with the available spectra of SNe IIP in the H $\alpha$ and He I $10830 \AA$ lines and estimate the wind density for particular SNe IIP ( $\S 3$. In $\S 4$ we address the issue of the H $\alpha$ absorption in the cool dense shell at the SN/CS interface of SNe IIP. We discuss implications of our models in the last section.

\section{MODEL}

The model for ejecta-wind interaction effects in $\mathrm{H} \alpha$ and He I $10830 \AA$ lines formed in the SN ejecta consists of three major parts: (i) an interaction model that provides the dynamical evolution of the $\mathrm{SN} /$ wind interface and the X-ray emission from the reverse and forward shocks; (ii) a model for the ionization and excitation of $\mathrm{H}$ and $\mathrm{He}$ in the unshocked SN ejecta irradiated by X-rays; and (iii) the calculation of line profiles. We perform (iii) using either a standard Sobolev approximation or the direct integration of the radiation transfer equation, depending on the validity of the Sobolev approximation.

\subsection{The interaction model}

The interaction of SN ejecta with CS wind leads to a canonical double-shock structure (Chevalier 1982a; Nadyozhin 1985) with the forward shock propagating in the CS gas and the reverse shock in the SN ejecta. We treat the CS interaction of ejecta in the thin shell approximation (Chevalier 1982b) in which the double-shock layer is reduced to an infinitely thin shell. We assume that the freely expanding $(v=r / t)$ SN envelope has a sharp boundary at the velocity $v_{\mathrm{c}}$ and begins to interact with a wind starting some moment $(t \sim 1$ day) which corresponds roughly to the shock breakout phase. Free expansion is expected to take several doubling times of the initial radius, or several days, to be set up, but the observations we are modeling are at later times. The maximum velocity $v_{\mathrm{c}}$ is set by the escape of radiation from the shock wave at shock breakout. 
For the density distribution $\rho(v)$ in the SN IIP envelope we use an analytical expression,

$$
\rho=\frac{\rho_{0}}{1+\left(v / v_{0}\right)^{k}},
$$

that closely approximates the combination of an inner plateau and outer power law tail found in hydrodynamic models (e.g., Utrobin 2007). The parameters $\rho_{0}$ and $v_{0}$ are determined by the kinetic energy $E$, ejecta mass $M$, and $k$ :

$$
M=4 \pi \rho_{0}\left(v_{0} t\right)^{3} C_{\mathrm{m}}, \quad E=\frac{1}{2} \frac{C_{e}}{C_{m}} M v_{0}^{2},
$$

where

$$
C_{\mathrm{m}}=\frac{\pi}{k \sin (3 \pi / k)}, \quad C_{\mathrm{e}}=\frac{\pi}{k \sin (5 \pi / k)} .
$$

The power law index $k$ lies in the range $k \sim 8-10$ and is henceforth set to be $k=9$. We adopt the boundary velocity for a typical SN IIP of $v_{\mathrm{c}}=1.5 \times 10^{4} \mathrm{~km} \mathrm{~s}^{-1}$, which is consistent with models of shock breakout (Matzner \& McKee 1999; Chevalier et al. 2006). Also, the blue edge of the $\mathrm{H} \beta$ emission in SN 1999gi on day 1 was observed to be at $15,000 \mathrm{~km} \mathrm{~s}^{-1}$ (Leonard et al. 2002b).

Hydrodynamical modeling predicts that at the shock breakout phase a thin dense shell forms at the outer boundary (Grasberg et al. 1971; Falk \& Arnett 1977; Chevalier 1981; Utrobin 2007). The physics of the 'boundary shell' formation is in the transition from the adiabatic to the radiative regime of shock wave propagation in the outermost layers of the exploding star. Simple considerations of radiative diffusion (Chevalier 1981) give an estimate for the shell mass:

$$
M_{s} \approx 2 \times 10^{-4}\left(\frac{R}{500 R_{\odot}}\right)^{2}\left(\frac{v_{b}}{10,000 \mathrm{~km} \mathrm{~s}^{-1}}\right)^{-1}\left(\frac{\kappa}{0.4 \mathrm{~cm}^{2} \mathrm{~g}^{-1}}\right)^{-1} M_{\odot},
$$

where $R$ is the progenitor star radius and $v_{b}$ is the velocity at shock breakout, before free expansion has been established. During the acceleration phase the boundary shell is subject to the Rayleigh-Taylor (RT) instability (Falk \& Arnett 1977) and therefore could be corrugated or even fragmented. However, it is not clear whether the RT instability results in full fragmentation; 2D or 3D hydrodynamic modeling of the phenomenon is lacking. We will treat the boundary shell in our model as an intact thin spherical shell which is already in place at the initial moment. The mass of the boundary shell is presumably equal to the mass of the outer envelope $\left(v>v_{\mathrm{c}}\right)$ with an extrapolated power law $\rho \propto v^{-k}$. This provides a good estimate of the shell mass for SN $1999 \mathrm{em}, \sim 3 \times 10^{-4} M_{\odot}$, in agreement with hydrodynamical modeling (Utrobin 2007). The boundary shell is considered below as a seed for the CDS that may, or may not, grow further due to radiative cooling at the reverse shock. 
To calculate the X-ray emission from the reverse and forward shocks we assume that the postshock layers are uniform and their densities are 4 times larger than the corresponding preshock density. We allow the electron temperature to be partially equilibrated, assuming the following interpolation:

$$
T_{e}=\max \left(0.1 T_{\mathrm{eq}}, \frac{t}{t+t_{\mathrm{eq}}} T_{\mathrm{eq}}\right),
$$

where $t_{\text {eq }}$ is the electron-ion temperature equilibration time (Spitzer 1962) and $T_{\text {eq }}$ is the equilibrium shock temperature with $T_{e}=T_{i}$. The lower limit $T_{e} / T_{\text {eq }}=0.1$ is indicated by observational data on supernova remnants with shock velocities of $\sim 1000 \mathrm{~km} \mathrm{~s}^{-1}$ (Rakowski) 2005). The reverse shock is equilibrated during the photospheric phase for a mass loss rate of $\sim 10^{-6} M_{\odot} \mathrm{yr}^{-1}$ with a wind velocity of $10 \mathrm{~km} \mathrm{~s}^{-1}$, while the forward shock is not. Since the reverse shock is equilibrated and dominates the X-ray luminosity, the uncertainty in $T_{e}$ does not affect our results. The shock X-ray luminosity is expressed in terms of the kinetic luminosity as $L_{\mathrm{X}}=\eta_{\mathrm{X}} L_{\text {kin }}$. The efficiency parameter $\eta_{\mathrm{X}}$ is smoothly interpolated between extreme cases, $\eta_{\mathrm{x}}=t /\left(t+t_{\mathrm{c}}\right)$, where $t_{\mathrm{c}}$ is the cooling time and is calculated for each shock using the cooling function of Sutherland \& Dopita (1993). The wind is assumed to be steady with the density $\rho \propto r^{-2}$. Since all the interaction effects for low wind velocities $(u)$ are determined by the wind density $\rho \propto \dot{M} / u$, we use hereafter the dimensionless wind density parameter $w=\dot{M}_{-6} / u_{10}$, where $\dot{M}_{-6}$ is the mass loss rate in units of $10^{-6} M_{\odot} \mathrm{yr}^{-1}$ and $u_{10}$ is the wind velocity in units of $10 \mathrm{~km} \mathrm{~s}^{-1}$.

The interaction model for a SN IIP with the wind density $w=1$ is illustrated in Fig. 1. The two cases shown have the same ejecta mass $M=19 M_{\odot}$ but different kinetic energy: $E=1.3 \times 10^{51} \mathrm{erg}$ and $E=0.65 \times 10^{51} \mathrm{erg}$. The high energy case corresponds presumably to SN 1999em (Utrobin 2007), while the low energy case illustrates SN 2004dj, as we will see below. Accordingly, we adopt $v_{\mathrm{c}}=15,000 \mathrm{~km} \mathrm{~s}^{-1}$ in the high energy case and $v_{\mathrm{c}}=13,000$ $\mathrm{km} \mathrm{s}^{-1}$ in the low energy case. The model with parameters for SN 1999em reproduces the observed (absorbed) Chandra X-ray luminosity of SN $1999 \mathrm{em}$ in the $0.5-8 \mathrm{keV}$ band (Pooley et al. 2002) for a distance $D=11.7 \mathrm{Mpc}$ (Leonard et al. 2003) quite satisfactorily (Fig. 1), which indicates that $w=1$ is a reasonable choice for the wind density. The estimated Galactic absorption, $N_{\mathrm{H}}=6 \times 10^{20} \mathrm{~cm}^{-2}$ (Pooley et al. 2002), is small and does not significantly affect the observed X-ray luminosity. The X-ray luminosity from the reverse shock dominates in both cases. The typical electron temperature for the reverse shock at $t>20 \mathrm{~d}$ is $\sim 1 \mathrm{keV}$, while for the forward shock it is $\sim 10 \mathrm{keV}$. The early behavior of the velocities differs from the self-similar evolution (Chevalier 1982b) because of the ejecta density cut-off in our model and the presence of the boundary shell at the initial moment. The mass of the boundary shell is $3 \times 10^{-4} M_{\odot}$ and $1.1 \times 10^{-4} M_{\odot}$ in the high and low energy models, respectively. The total mass of the CDS attained at the time of the termination 
of the radiative regime in the reverse shock $\left(t_{\mathrm{rad}}\right)$ is $6 \times 10^{-4} M_{\odot}$ and $3.2 \times 10^{-4} M_{\odot}$ with $t_{\mathrm{rad}}=21 \mathrm{~d}$ and $t_{\mathrm{rad}}=18 \mathrm{~d}$ for the high and low energy models, respectively. Remarkably, the reverse shock is adiabatic in the self-similar models for the same parameters through this epoch. This fact is related to the different dynamics of the self-similar model at an early stage, which results in a lower preshock density at the reverse shock.

The spectrum of X-rays is described below using a standard approximation $F_{E} \propto$ $E^{-q} \exp \left(-E / T_{e}\right)$. We adopt $q=0.4$ for the forward shock, a reasonable approximation for temperature $\sim 10 \mathrm{keV}$ ( $\mathrm{Cox} 2000)$, while for the low temperature reverse shock we take $q=1$. The latter choice qualitatively describes the calculated energy distribution of X-rays with a significant contribution of emission lines for the electron temperature $T_{e}=1 \mathrm{keV}$ (Nymark et al. 2006).

\subsection{Model of $\mathbf{H}$ and $\mathrm{He}$ excitation}

The hydrogen and helium ionization in a SN IIP atmosphere at the photospheric epoch is time-dependent (Utrobin \& Chugai 2002, 2003, 2005). To take this effect into account, we use a hydrogen atom represented by four levels and continuum. All the essential radiative and collisional processes for hydrogen are taken into account, including non-thermal ionization and excitation induced by X-ray absorption. The excitation of the helium $2^{3} \mathrm{~S}$ level is determined by a simple model with two excited levels $\left(2^{1} \mathrm{~S}\right.$ and $\left.2^{3} \mathrm{~S}\right)$. We consider only major processes for the population of the $2^{3} \mathrm{~S}$ level: nonthermal excitation and ionization with subsequent recombination into triplet states, depopulation by the collisional deexcitation transition $2^{3} \mathrm{~S}-2^{1} \mathrm{~S}$, Penning ionization (Chugai 1991) and continuum absorption in the $10830 \AA$ line $\left(2^{3} \mathrm{~S}-2^{3} \mathrm{P}\right.$ transition) with the subsequent spontaneous transition $2^{3} \mathrm{P}-1^{1} \mathrm{~S}$ terminated by photon escape. The energy balance includes the energy deposition due to the absorption of X-rays and the hydrogen photoionization from excited levels by photospheric radiation. The cooling includes adiabatic loss ( $p d V$ work), free-free, free-bound, boundbound emission of hydrogen, and cooling in the [O I] 6300, $6364 \AA$ and Mg II $2800 \AA$ lines. The Fe II line cooling is approximately included by multiplying the Mg II $2800 \AA$ cooling rate by a factor of two. Given the possibility of a high temperature regime $\left(T_{e}>20,000 \mathrm{~K}\right)$ in the outer layers, the cooling at this range of temperatures is treated using the cooling function of Sutherland \& Dopita (1993).

The distribution of the ionization and excitation of $\mathrm{H}$ and $\mathrm{He} \mathrm{I}$ in the atmosphere at one time is calculated by tracking the evolution of a Lagrangian zone after its emergence from the photosphere. The initial ionization and excitation at the photosphere is set assuming SahaBoltzmann equations for the effective temperature. Subsequent ionization and excitation are 
computed by solving the system of time-dependent kinetic equations together with the timedependent energy balance. The average intensity of the photospheric radiation is assumed to be $J_{\nu}=W I_{\nu}$, where $W$ is the dilution factor and $I_{\nu}$ is the photospheric brightness. The luminosity evolution at the photospheric epoch $(t<100 \mathrm{~d})$ is described by the expression

$$
L=L_{0}\left[1+\exp \left(-t / t_{1}\right)\right]
$$

With $L_{0}=10^{42} \mathrm{erg} \mathrm{s}^{-1}$ and $t_{1}=10 \mathrm{~d}$, this corresponds to the bolometric luminosity of SN 1999em (Elmhamdi et al. 2003), assuming a distance of $11.7 \mathrm{Mpc}$. The photospheric radius is determined by the velocity at the photosphere and the age $\left(r_{\mathrm{p}}=v_{\mathrm{p}} t\right)$, where $v_{\mathrm{p}}$ is deduced from observational data of Leonard et al. (2002a). The spectrum of the photospheric continuum is taken to be blackbody with $T=T_{\text {eff }}$ in the wavelength region longward of the Balmer jump, while the ultraviolet (UV) radiation shortward of the Balmer continuum edge is suppressed by a factor $f_{\mathrm{UV}} \leq 1$ which was assumed to monotonically drop after the explosion from unity to 0.03 on a timescale of $10 \mathrm{~d}$. This evolution was deduced from the behavior

observed in SN 1987A (Pun et al. 1995). We found that the results are only weakly sensitive to the specific form of the variation of $f_{\mathrm{UV}}$. The energy deposition rate in the atmosphere due to the absorption of X-rays is calculated with an absorption coefficient $k_{\mathrm{X}}=100 E^{-8 / 3}$ $\mathrm{cm}^{2} \mathrm{~g}^{-1}$ (where $E$ is in $\mathrm{keV}$ ). The deposited energy is shared between heating, ionization, and excitation according to the recipes of Kozma \& Fransson (1992) and Xu et al. (1992).

The solution of the kinetic equations for each Lagrangian zone provides populations of the hydrogen levels $n_{2}$ and $n_{3}$, and the $2^{3} \mathrm{~S}$ level of He I in the atmosphere. These values are then used to calculate line profiles of $\mathrm{H} \alpha$ and He I $10830 \AA$. We compared our model in the case of zero wind density with the detailed model of Utrobin \& Chugai (2005) and found that the present model results in slightly weaker $\mathrm{H} \alpha$ absorption; at the relative intensity of 0.9 , the radial velocity in the simple model is lower by $\sim 500 \mathrm{~km} \mathrm{~s}^{-1}$. This drawback of the simple model does not noticeably affect the amplitude of the calculated HV absorption formed in the external layers, where the ionization unaffected by X-ray absorption is small.

\section{HIGH VELOCITY FEATURES IN H AND He LINES}

\subsection{Model properties}

The model line profiles of $\mathrm{H} \alpha$ and He I $10830 \AA$ are computed for different wind densities, ages, ejecta mass and energy (Table 1 ). The standard model is characterized by $M=19 M_{\odot}$, $E=1.3 \times 10^{51} \mathrm{erg}, k=9$. In all cases $v_{\mathrm{c}}=15,000 \mathrm{~km} \mathrm{~s}^{-1}$ with the exception of the very low energy model $(\mathrm{eW})$ for which $v_{\mathrm{c}}=6500 \mathrm{~km} \mathrm{~s}^{-1}$. We designate a model at a particular time by adding the age in days to the model name; e.g., W50 stands for model W on day 
50. The effect of the wind density on $\mathrm{H} \alpha$ on day 50 is shown in Fig. $2 a$ for the wind density parameters $w=0.5,1,2$, and 4 . Without a wind, the $\mathrm{H} \alpha$ absorption on day 50 forms because of the time-dependent ionization effect; a high ionization is maintained due to Ly $\alpha$ trapping and reionization from excited levels of hydrogen. The CS interaction results in the ionization and excitation of the outer recombined layers of unshocked ejecta and, as a result, a HV absorption feature appears in the blue wing of the undisturbed profile. Fig. 3 illustrates how $\mathrm{H} \alpha$ absorption is modified by the CS interaction. For $w=0.5$, the CS interaction effect is small (Fig. 2 $a$ ) and the wind density $w=0.5$ seems to be the least that could be detected by $\mathrm{HV}$ absorption in $\mathrm{H} \alpha$. For $w \sim 1$, the strength of the $\mathrm{HV}$ absorption increases with the wind density roughly as $w^{2}$ and for $w>2$ becomes saturated. The decrease of the velocity of the blue absorption edge is due to the deceleration caused by the dense wind. The fact that for $w=4$ the HV absorption merges with undisturbed $\mathrm{H} \alpha$ absorption implies that in the case of a very dense wind it would be difficult to infer the density parameter $w$ using only the $\mathrm{H} \alpha$ absorption.

The evolution of the HV absorption (Fig. 2b) shows that there is an optimal phase at about the middle of the plateau $(\sim 50 \mathrm{~d})$ when the CS interaction effect is most pronounced. At an early stage (e.g., on day 20) the CS interaction effect is not clearly seen because of the merging of $\mathrm{HV}$ absorption with the strong undisturbed absorption, while at a late stage (e.g., 80 d) HV absorption becomes very faint. The sensitivity of HV absorption to the ejecta density is shown by the model mW50 with a mass $10 M_{\odot}$ and energy $9 \times 10^{50} \mathrm{erg}$. The energy is chosen to match the blue wings of the main absorption of models mW50 and W50. The HV absorption in the low mass case turns out the same as in the model W50, i.e., the effect of reducing the ejecta density by a factor of 2 is negligible. We also consider the CS interaction effect for SNe IIP with a very low kinetic energy, $E=3 \times 10^{50} \mathrm{erg}$, which presumably applies to the low-luminosity SNe IIP, e.g., SN 1999br (Pastorello et al. 2004). This model with $v_{\mathrm{c}}=6500 \mathrm{~km} \mathrm{~s}^{-1}$ and $w=1$ predicts strong $\mathrm{HV}$ absorption which is merged with the undisturbed absorption (Fig. 2d). For all the models when HV absorption is unsaturated, the Sobolev optical depth of this feature for $w \sim 1$ is $\tau \leq 1$. This implies that in the $\mathrm{H} \beta$ line the optical depth $\leq 0.14$, so we do not expect that $\mathrm{HV}$ absorption in this line will be pronounced (but see $\S$ 4).

We examined the sensitivity of HV absorption to the power law index $q$ of the X-ray spectrum of the reverse shock. We found that a softer spectrum $(q=1.2)$ and harder spectrum $(q=0.8)$ produce slightly deeper HV absorption, so the preferred case $q=1$ corresponds approximately to a minimal effect of the CS interaction on $\mathrm{H} \alpha$. We checked the sensitivity of HV absorption to the adopted SN bolometric luminosity as well. The HV absorption strength anticorrelates with the bolometric luminosity, but the dependence is weak. The mechanism for this dependence is the depopulation of excited levels by photoionization. 
The CS interaction effect in the He I $10830 \AA$ line substantially differs from that in $\mathrm{H} \alpha$ in one important respect: in the absence of a wind the model does not predict noticeable absorption in HeI $10830 \AA$ (Fig. 4a), except for a very early stage $(t<10 \mathrm{~d}$ ). The wind density $w=0.5$ produces weak HV absorption, on the verge of detectability, while the case $w=1$ yields $\mathrm{HV}$ absorption with a relative depth of $\sim 0.2$ and could be easily detected. The absorption gets markedly stronger for larger $w$, showing the sensitivity of HeI absorption to the wind density in this particular range of the $w$ parameter. Remarkably, while in $\mathrm{H} \alpha$ the contribution of the interaction effect for $w=4$ is difficult to discern (Fig. $2 a$ ), the He I $10830 \AA \mathrm{HV}$ absorption provides a probe for such a dense wind.

The strength of HV absorption in He I $10830 \AA$ increases between days 20 and 50 but gets weak on day 80 (Fig. 4b), resembling the behavior of $\mathrm{HV}$ absorption in $\mathrm{H} \alpha$. The effects of the ejecta density (Fig. 4 $c$ ) are similar to those in $\mathrm{H} \alpha$. The $\mathrm{HV}$ absorption for the low energy case is very strong and easily discernable, unlike in $\mathrm{H} \alpha$.

\subsection{Comparison with observations}

\subsection{1. $H V$ absorption in $H \alpha$}

Spectra of SN 1999em (Elmhamdi et al. 2003) on days 41 and 54 (assuming the explosion date JD 2451476) show a depression in the blue wing of the $\mathrm{H} \alpha$ line (Fig. 51) at the predicted position of $\mathrm{HV}$ absorption $\left(-10,000\right.$ to $\left.-12,000 \mathrm{~km} \mathrm{~s}^{-1}\right)$. To model the $\mathrm{H} \alpha$ profiles we adopted the standard parameters $M=19 M_{\odot}, E=1.3 \times 10^{51} \mathrm{erg}, k=9$, and $v_{\mathrm{c}}=15,000$ $\mathrm{km} \mathrm{s}^{-1}$. We find the best value of the wind density parameter is $w=1$ with an uncertainty of $\sim \pm 0.07$, assuming the other parameters to be fixed. The indicated error reflects only the accuracy of estimating this parameter from the best fit and does not include errors in the observed spectrum and uncertainties in the model. Also on day 54, the model reproduces the general strength of the observed HV absorption (Fig. 5). Yet we find that the observed HV absorption on day 54 seems to contain a notch component that is deeper than in the model.

The question arises of why the model fails to describe in detail the undisturbed $\mathrm{H} \alpha$. The model emission is weaker, while the absorption is stronger, than is observed (Fig. 5 and Utrobin 2007). The weak model emission is probably related to our crude description of the UV continuum in the SN atmosphere (cf. Utrobin \& Chugai 2005). However, this cannot explain why the observed absorption is shallow. In fact, a check of available spectra of SNe IIP indicates that the $\mathrm{H} \alpha$ absorption seems to be shallow in any normal SN IIP (e.g., SN 2004dj, see below). In contrast, for the peculiar SN 1987A related to the explosion of 
a blue supergiant, the $\mathrm{H} \alpha$ absorption is deep and well reproduced by the time-dependent model (Utrobin \& Chugai 2005). We speculate that this is a strong indication that the shallowness of the $\mathrm{H} \alpha$ absorption in normal SNe IIP is related to the red supergiant structure of the presupernova. For instance, convection in the red supergiant atmosphere could produce large amplitude density perturbations that are significantly amplified during the blast wave propagation. As a result, the outer layers of ejecta would acquire a clumpy structure responsible for the shallowness of the $\mathrm{H} \alpha$ absorption.

Returning to the HV absorption, in Fig. 6] we show the model fit to the observed $\mathrm{H} \alpha$ in SN 2004dj (Korcakova et al. 2005) on days 55 and 64 (for an explosion date JD 2453185). The model parameter set is $M=19 M_{\odot}, k=9$, as for SN $1999 \mathrm{em}$, but $E=6.7 \times 10^{50}$ erg. One could retain the energy $1.3 \times 10^{51} \mathrm{erg}$ used for SN $1999 \mathrm{em}$ and vary $M$. However, in this case one requires $M \approx 50 M_{\odot}$ to attain a comparable fit. Since the ejecta mass unlikely exceeds $20 M_{\odot}$, the lower energy case for SN 2004dj is preferred. In accord with the lower energy we take $v_{\mathrm{c}}=13,000 \mathrm{~km} \mathrm{~s}^{-1}$ and lower velocities at the photosphere by a factor 0.9 compared to SN $1999 \mathrm{em}$. The derived wind density $(w=1.27 \pm 0.1)$ is determined by the strength of the HV absorption and its position, while the lower energy is needed to reproduce the correct position of the HV absorption. To check the effect of the adopted boundary velocity $v_{\mathrm{c}}$, we modeled the case $v_{\mathrm{c}}=11,000 \mathrm{~km} \mathrm{~s}^{-1}$. In this case the wind density is lower, $w=0.9$. We prefer $v_{\mathrm{c}}=13,000 \mathrm{~km} \mathrm{~s}^{-1}$ on the basis of the arguments presented below in Sec. 4.2 ,

On day 55 the HV absorption in the observed spectrum has the appearance of a relatively broad shallow depression similar to SN $1999 \mathrm{em}$ on day 41. A bit later, on day 64, a notch ("V-shaped" narrow absorption) appears at a radial velocity $\sim-8200 \mathrm{~km} \mathrm{~s}^{-1}$ (Fig. 66). The feature dominates the HV absorption until at least day 102 in spectra of SN 2004dj reported by Vinko et al. (2006). Neither the strength nor the shape of the notch at the late photospheric stage $t \geq 60 \mathrm{~d}$ can be produced by a model in which the HV absorption forms only in the unshocked ejecta. This suggests that the notch in $\mathrm{H} \alpha$ has a different origin which is addressed in $\S$. 4 .

The HV absorption in SN 2004dj has a markedly lower velocity compared to SN 1999em at a similar epoch $\left(8200\right.$ vs. $\left.11,500 \mathrm{~km} \mathrm{~s}^{-1}\right)$. This fact is significant for the origin of the depression in the blue wing of $\mathrm{H} \alpha$ since it rules out the possibility that the feature might be produced by some unidentified metal line. Photospheric velocities at this epoch are similar in both SNe within several $100 \mathrm{~km} \mathrm{~s}^{-1}$, so the expected positions of a metal line absorption should coincide in both SNe within the same range of velocities. Since this is not the case, we conclude that $\mathrm{HV}$ absorption in these supernovae cannot be produced by an unidentified line. An additional argument against the 'metal line' option is that SN 1987A does not show 
$\mathrm{HV}$ absorption in $\mathrm{H} \alpha$ at all. This would be difficult to understand in the context of the metal line option, but is easily explained by the very low density of the pre-SN wind $\left(w \sim 2 \times 10^{-3}\right.$, Chevalier \& Dwarkadas 1995) that is incapable of producing detectable interaction effects.

\subsection{2. $H V$ absorption in HeI $10830 \AA$}

The HV absorption of He I $10830 \AA$ in SN 1999em on day 20 was identified by Elmhamdi et al. (2003) and attributed originally to radioactive excitation by external ${ }^{56} \mathrm{Ni}$. Here we abandon this explanation and propose excitation of He in the external layers by CS interaction. The He I $10830 \AA$ line of SN 1999em on day 20 (Elmhamdi et al. 2003) is shown in Fig. 7 together with models for $w=1$ and the best value $w=1.15$, which has an uncertainty of \pm 0.07 . The wind density parameter derived from the HV absorption strength in HeI $10830 \AA$ agrees well with the value $w=1$ found from the $\mathrm{H} \alpha$ line. The clear-cut observed profile and the sensitivity of HV absorption of HeI $10830 \AA$ to the wind density makes this line a valuable diagnostic tool for probing the CS wind in SNe IIP.

Interestingly, based on a time-dependent model for a SN IIP spectrum without CS interaction, Dessart \& Hillier (2007) predict strong HV absorption in He I $10830 \AA$ at a relatively late epoch; on day 50 , the relative depth of HeI $10830 \AA$ is $\approx 0.1$ for an enhanced He abundance $n(\mathrm{He}) / \mathrm{n}(\mathrm{He})=0.2$. This suggests a relative depth of $\approx 0.05$ for a solar He abundance. The absorption lies in the range of $-12,000$ to $-17,000 \mathrm{~km} \mathrm{~s}^{-1}$, which corresponds to the blue wing of HeI $10830 \AA$ in SN 1999em on day 20 (Fig. 7). The HeI $10830 \AA$ line in SN $1999 \mathrm{em}$ on day 20 does not show evidence for the additional He absorption in the blue wing. Yet it is possible that in a more detailed model the time-dependent effects for He lines might be larger than in our simple model and would substantially contribute to the HV absorption of the He I $10830 \AA$ line at an age $t>20$ d. However, for SN 1999em on day 20, we note the close correspondence between the $w$ values deduced from the $\mathrm{H} \alpha$ and HeI $10830 \AA$ lines.

Spectra of SN 1999em covering the He I $10830 \AA$ line are also reported by Hamuy et al. (2001) for three epochs: 8, 24, and $34 \mathrm{~d}$. The first spectrum shows a strong HeI $10830 \AA$ line with a P Cygni profile. This line, related to the early high temperature phase, rapidly disappears and is not seen in the next two spectra. Instead, HV absorption appears in this line. On day 24, the HV absorption of He I $10830 \AA$ is much the same as on day 20 in Fig. 7 , and it also appears on day 34. A steady HV absorption feature with time is expected in our model (4b). On day 34, an additional redder absorption at $\sim 10549 \AA$ appears separate from the HV absorption. The transformation of the He I $10830 \AA$ line from a single HV absorption into a double absorption structure is also observed between days 22 and 44 in another Type 
IIP supernova, SN 1995V (Fassia et al. 1998). We believe that the low velocity absorption is the result of He excitation by gamma-ray photons leaking from the inner radioactive decays of ${ }^{56} \mathrm{Ni}$ and ${ }^{56} \mathrm{Co}$ (Fassia et al. 1998; Chugai 1987). To produce the required excitation of He about $\sim 10^{-5} M_{\odot}$ of ${ }^{56} \mathrm{Ni}$ should be mixed out to a velocity $\sim 4000 \mathrm{~km} \mathrm{~s}^{-1}$, which does not contradict the conclusion that the majority of the ${ }^{56} \mathrm{Ni}$ resides in deeper layers with velocity $\leq 600 \mathrm{~km} \mathrm{~s}^{-1}$ (Utrobin 2007).

\section{ORIGIN OF THE NOTCH IN H $\alpha$}

We now address the issue of the notch that emerged in $\mathrm{H} \alpha$ in both SN 1999em and SN 2004dj at about day 60. We propose that the notch is produced by $\mathrm{H} \alpha$ absorption of the photospheric radiation in the CDS. We also consider an alternative mechanism: additional excitation of hydrogen in the unshocked ejecta by Ly $\alpha$ photons emitted from the CDS.

\subsection{CDS vs. unshocked ejecta}

We first study the preferred explanation for the notch, $\mathrm{H} \alpha$ absorption in the CDS, and consider the clearly observed case of SN 2004dj. In modeling the $\mathrm{H} \alpha$ absorption produced by the CDS, the principal parameters are the column density of the cool gas, and its excitation temperature and turbulent velocity. The undisturbed or weakly disturbed CDS is characterized by a turbulent velocity of the order of the thermal velocity of the cool gas, $\sim 10-20$ $\mathrm{km} \mathrm{s}^{-1}$. However, with this turbulent velocity the equivalent width of the $\mathrm{HV}$ absorption turns out to be too small. A possible solution is to increase the turbulent velocity, as expected by the action of the Rayleigh-Taylor (RT) instability of the decelerating thin shell. A significant deceleration of the model CDS of SN 2004dj, about 20\% during the first 10 days (see low energy case in Fig. 1), favors the development of the RT instability of the CDS followed by mixing of the cool dense gas with hot forward shock gas (Chevalier \& Blondin 1995; Blondin \& Ellison 2001). The mixed cool gas thus can acquire a significant velocity dispersion and turbulent velocities up to $\sim 0.1$ of the shell velocity. Both components could contribute to the notch formation: absorption in the weakly disturbed CDS and in the mixed CDS material. We therefore consider two components: (1) a weakly perturbed spherical shell with a turbulent velocity of $u_{\mathrm{t}} \approx 20 \mathrm{~km} \mathrm{~s}^{-1}$ and (2) mixed CDS gas with a large turbulent velocity $u_{\mathrm{t}} \approx 400-500 \mathrm{~km} \mathrm{~s}^{-1}$. The mass fraction of the second component is denoted $\beta$. The corresponding spectral components of the HV absorption related to the CDS can be dubbed narrow notch and broad notch. These components should be distinguished from the 'ejecta component' - a broader shallow HV absorption formed in the unshocked ejecta 
and addressed in the previous section. A cartoon (Fig. 8) illustrates the origin the HV absorption produced by the ejecta and the CDS.

Hydrogen excitation of the CDS gas is presumably maintained by the absorption of $\mathrm{X}$-rays emitted from the reverse shock. The absorbed energy of X-rays is assumed to be homogeneously distributed through the CDS material, as expected for the modest shell column densities for our situation. The number density in the CDS is determined by pressure equilibrium $P \approx \rho_{\mathrm{w}} v_{\mathrm{s}}^{2}$ (where $\rho_{\mathrm{w}}$ is the pre-shock wind density) for the typical temperature of the cool gas of $\sim 10^{4} \mathrm{~K}$ implied by the energy balance. On day 64 , the CDS mass in our model for SN $2004 \mathrm{dj}$ is $3.2 \times 10^{-4} M_{\odot}$. The other input parameters provided by the interaction model are: CDS radius, $R_{\mathrm{s}}=5.2 \times 10^{15} \mathrm{~cm}$, velocity $v_{\mathrm{s}}=8350 \mathrm{~km} \mathrm{~s}^{-1}$, X-ray luminosity of the reverse shock $L_{\mathrm{X}}=1.53 \times 10^{38} \mathrm{erg} \mathrm{s}^{-1}$, and electron temperature in the reverse shock $k T_{\mathrm{X}}=1.36 \mathrm{keV}$. The population of the second level of hydrogen is computed using a steady-state approximation (justified since $n_{e}>10^{8} \mathrm{~cm}^{-3}$ ), and assuming a two level plus continuum model for the hydrogen atom. Both non-thermal excitation and ionization by secondary electrons lead to the excitation of the second level, while depopulation proceeds by the two-photon transition, Ly $\alpha$ escape and collisional deexcitation.

The calculated profiles of HV absorption related to the CDS are overplotted on the model $\mathrm{H} \alpha$ profile with ejecta HV absorption and displayed in Fig. 9 along with the observed spectrum of SN 2004dj (Korcakova et al. 2005) on day 64. Shown are three cases: one without the CDS absorption and two cases with the CDS absorption that differ by the mass fraction of the broad CDS component $(\beta=0.1$ and 0.5$)$. For the quoted values, the optical depths of the narrow components are 520 and 175, while these values for the broad component are 0.2 and 0.45 , respectively.

The absolute value of the radial velocity of the HV absorption produced by the CDS $\left(v_{\mathrm{a}}\right)$ is slightly lower than the velocity of the CDS, $v_{\mathrm{s}}$, owing to the sphericity effect. Assuming uniform brightness of the photosphere (Lambert's law) it is straightforward to show that the average absolute value of the radial velocity of the absorption is

$$
v_{\mathrm{a}}=\frac{2}{3} \frac{v_{\mathrm{s}}}{x^{2}}\left[1-\left(1-x^{2}\right)^{3 / 2}\right]
$$

where $x=v_{\mathrm{p}} / v_{\mathrm{s}}$ is the ratio of the photospheric to the CDS velocity. According to this expression $v_{\mathrm{a}}<v_{\mathrm{s}}$ and, in the limit $x \ll 1, v_{\mathrm{a}}=v_{\mathrm{s}}$ with high accuracy. The sphericity effects are responsible also for the additional broadening of the absorption over the radial velocity range $\left(v_{\mathrm{s}}^{2}-v_{\mathrm{p}}^{2}\right)^{1 / 2}<\left|v_{r}\right|<v_{\mathrm{s}}$, which is $\sim 460 \mathrm{~km} \mathrm{~s}^{-1}$ for the model of SN 2004dj on day 64 .

The alternative mechanism is that the notch could form as a result of an enhancement of hydrogen excitation in the unshocked SN ejecta due to the scattering of Ly $\alpha$ photons emitted from the CDS that in turn is irradiated by X-rays from the reverse shock. A 
resonance condition implies that Ly $\alpha$ photons can scatter in the unshocked ejecta only in the narrow velocity range $v_{\mathrm{s}}<v<v_{\mathrm{sn}}$, where $v_{\mathrm{sn}}$ is the boundary velocity of the unshocked $\mathrm{SN}$ ejecta. The expected flux of Ly $\alpha$ from the CDS is

$$
F_{12}=\frac{1}{2} \psi \frac{L_{\mathrm{X}, \mathrm{abs}}}{4 \pi R_{\mathrm{s}}^{2}},
$$

where $\psi$ is the efficiency of Ly $\alpha$ emission and $L_{\mathrm{X} \text {,abs }}$ is the X-ray luminosity absorbed by the CDS. The average excitation rate in the ejecta within the velocity width $\Delta v=v_{\mathrm{sn}}-v_{\mathrm{s}}$ is balanced by Ly $\alpha$ escape; other depopulation mechanisms are negligible. The population of the second level $\left(n_{2}\right)$ thus is approximately determined by the rate equation

$$
n_{2} A_{21} \beta_{12}=\frac{F_{12}}{h \nu_{12} \Delta v t}
$$

where $t$ is the SN age, $h \nu_{12}$ is the Ly $\alpha$ photon energy, and $\beta_{12}$ is the Sobolev escape probability for Ly $\alpha$. For the model of SN 2004dj, the notch in $\mathrm{H} \alpha$ produced by Ly $\alpha$ scattering in the ejecta is calculated on day 64 (Fig. 9). Two cases, with efficiencies $\psi=0.1$ and 0.5 , are plotted. One sees that even for the unacceptably high efficiency $\psi=0.5$ the notch produced by the Ly $\alpha$ scattering is significantly weaker than that predicted by the absorption in the CDS. We conclude that the latter mechanism is the most likely explanation of the notch in $\mathrm{H} \alpha$.

We examined whether the absorption in the CDS might produce some effect in the He I $10830 \AA$ line. For the above model, the optical depth in the HeI $10830 \AA$ line turns out to be a factor of $\sim 10^{2}$ lower than in $\mathrm{H} \alpha$. Therefore, it is unlikely that the notch can be seen in HeI $10830 \AA$. The main reason is the efficient depopulation of the $2^{3} \mathrm{~S}$ level in the CDS by Penning ionization of hydrogen.

Given the relatively high $\mathrm{H} \alpha$ optical depth of the CDS, a notch at the same velocity could be seen in $\mathrm{H} \beta$ as well. We inspected spectra of SN 1999em (Leonard et al. 2002a) and found the signature of a notch in $\mathrm{H} \beta$ at the right position $\left(\approx-11,500 \mathrm{~km} \mathrm{~s}^{-1}\right)$ between days 39 and 81. In fact, Leonard et al. (2002a) already discussed the presence of the HV absorption in $\mathrm{H} \alpha$ and $\mathrm{H} \beta$ in spectra of SN 1999em and concluded that these features cannot be caused by metal line absorptions. We also find a notch in $\mathrm{H} \beta$ in spectra of SN 2004dj obtained by Vinko et al. (2006). Since the HV absorption components of $\mathrm{H} \alpha$ and $\mathrm{H} \beta$ have not been previously mentioned for this supernova, we show parts of the SN 2004dj spectra (Vinko et al. 2006) retrieved from the SN archive SUSPECT (Fig. 10). The spectra show conspicuous notches at about $-8000 \mathrm{~km} \mathrm{~s}^{-1}$ both in $\mathrm{H} \alpha$ and $\mathrm{H} \beta$ between days 67 and 102 . Below we will model HV absorption in both lines in more detail. 


\subsection{Rayleigh-Taylor mixing effects in $\mathrm{HV}$ absorption}

Here we present a model that can account for the strength and shape of the notch in $\mathrm{H} \alpha$ of SN 2004dj at the late photospheric epoch. Preliminary modeling suggests that the simple two-component model of the CDS (narrow and broad) introduced above is not able to describe the strength of the notch at late times $(\sim 100 \mathrm{~d})$ : the excitation produced by the X-ray absorption is insufficient. This suggests the presence of a more efficient excitation mechanism or an additional source of heat. Another problem is the relative depth of notches in the $\mathrm{H} \alpha$ and $\mathrm{H} \beta$ lines: they are comparable and shallow. This indicates that some veiling is present, possibly due to clumpiness. Both reasons force us to modify the two-component model of the CDS absorption.

An important outcome of the RT mixing of the CDS material in the forward shock is the growth of the area of the contact surface $(S)$ between cool and hot gas. The development of mixing may be thought as the progressive decrease of the minimum linear scale of fragments $(\lambda)$. This process is demonstrated in numerous experiments on the RT instability and in the case of SN/CS interaction is clearly seen in 2D and 3D hydrodynamical computations (Blondin \& Ellison 2001). A fragmentation cascade of a fixed amount of cool gas leads to the scaling $S \propto 1 / \lambda$, while for a stationary mixing process $S \propto \lambda^{-1 / 3}$ (Sreenivasan et al. 1989). The growth of $S$ and, accordingly, of the surface-to-volume ratio for the mixed cool gas, should lead to a greater role of thermal conduction in the heating of the cool gas at late times. We speculate that this is the missing factor that could resolve the excitation problem.

To describe the effect of thermal conduction, we introduce a third CDS component: an additional broad CDS component powered predominantly by thermal conduction. The mass fraction of the third component is $\mu \ll 1$, while the cumulative area of the contact surface for the third component, $S=C_{\mathrm{s}} 4 \pi R_{\mathrm{s}}^{2}$, can be large, with an area ratio $C_{\mathrm{s}} \gg 1$. We assume that the third component is homogeneously heated by thermal conduction, which is a sensible approximation for a small thickness of sheets of CDS gas, $\sim 10^{9} \mathrm{~cm}$, comparable to the mean free pass length of hot electrons and protons in the cool dense gas. The heat flux is presumably a small fraction of the saturated flux $F_{\mathrm{c}}=\eta_{\mathrm{c}} F_{\text {sat }}$; we adopt $\eta_{\mathrm{c}}=0.01$ to allow qualitatively for the possible suppression of thermal conduction by a magnetic field. The saturated flux is taken of the form $F_{\text {sat }}=0.4\left(2 k T_{e} / \pi m_{e}\right)^{1 / 2} n_{e} k T_{e}$ (Cowie \& McKee 1977). The forward shock is not equilibrated $\left(T_{e} \ll T_{i}\right)$, so the ion (proton) conduction could be significant. In our case, the ratio of proton-to-electron thermal flux is $\left(T_{i} / T_{e}\right)^{3 / 2}\left(m_{e} / m_{p}\right)^{1 / 2} \sim$ 2 and this fact is taken into account. Ionization and thermal balance are solved for the dense hydrogen cooled by Ly $\alpha$ and two-photon radiation.

The RT mixing generally produces an inhomogeneous (lumpy) distribution of mixing regions. This is the result of the existence of a dominant angular scale of the RT instability, 
about $10^{\circ}-15^{\circ}$ Chevalier \& Blondin 1995; Blondin \& Ellison 2001) and of the intermittence of the turbulent mixing. We describe the distribution of mixed regions as an ensemble of isolated lumps which occupy a fraction $f \sim 0.1-1$ of the volume; $f=1$ corresponds to a homogeneous mixture of cool and hot phases. The fraction $f$ should not be confused with the filling factor of the mixed cool dense gas, which is of the order of $10^{-5}$. Observationally, the lumpiness of mixing zones could be manifested as a veiling effect, i.e., a comparable moderate depth of absorption lines with different optical depth (viz., $\mathrm{H} \alpha$ and $\mathrm{H} \beta$ as in our case). To facilitate the treatment of the radiation transfer in the lumpy medium we use an occultation optical depth, $\tau_{\text {oc }}$, i.e., the average number of lumps along the radius. This value is defined by $f$ and a ratio of the total thickness of the mixing layer (forward post-shock layer $\Delta R$ ) to the mean intercepted length of the lump $d$ (for a sphere, $d=\frac{4}{3} \times$ radius): $\tau_{\text {oc }}=f(\Delta R / d)$. Generally, $\tau_{\text {oc }}$ could be different for each of the three components. However, we assume the same value of $\tau_{\text {oc }}$ for all three components. The effective optical depth in the line $(\tau)$ then can be expressed through the average optical depth of homogeneously distributed CDS material $\tau_{\text {av }}$ and $\tau_{\text {oc }}: \tau=\tau_{\text {oc }}\left[1-\exp \left(\tau_{\text {av }} / \tau_{\text {oc }}\right)\right]$ (Chugai \& Chevalier 2006). We thus consider a three component model of the cool dense gas which includes: (i) a narrow CDS component with turbulent velocity $u_{\mathrm{t}} \approx 20 \mathrm{~km} \mathrm{~s}^{-1}$, (ii) a broad CDS component with $u_{\mathrm{t}} \approx 400-500$ $\mathrm{km} \mathrm{s}^{-1}$, both powered by X-rays, and (iii) a broad CDS component powered by the thermal conduction.

The CDS notches in $\mathrm{H} \alpha$ and $\mathrm{H} \beta$ in SN 2004dj are calculated on the basis of the previous SN 2004dj model assuming $u_{\mathrm{t}}=20 \mathrm{~km} \mathrm{~s}^{-1}$ for narrow and $u_{\mathrm{t}}=400 \mathrm{~km} \mathrm{~s}^{-1}$ for broad CDS components. The adopted fraction of the broad CDS component is $\beta=0.5$ at the early epoch $(t=67 \mathrm{~d})$, with a subsequent increase proportional to the age, while the fraction of the third component is $\mu=0.1$ (Table 2). The CDS absorption in $\mathrm{H} \alpha$ is superimposed on the model line profile with the ejecta $\mathrm{HV}$ absorption. For the $\mathrm{H} \beta$ line, the CDS HV absorption is superimposed on the background spectrum taken as a linear interpolation between the blue and red wings of HV absorption in the observed profile. The occultation optical depth $\tau_{\text {oc }} \sim 0.15-0.4$ (Table 2 ) is chosen to satisfy the ratio $\mathrm{H} \alpha / \mathrm{H} \beta$, while the area ratio $C_{\mathrm{s}}$ is determined from the strength of the absorption. Fig. (11) shows that a three-component model with the area ratio $C_{\mathrm{s}} \sim 8-30$ (Table 2) describes the $\mathrm{HV}$ absorption of both $\mathrm{H} \alpha$ and $\mathrm{H} \beta$ lines satisfactorily. On day 102 the ejecta HV absorption is somewhat stronger, which is responsible for the red-blue asymmetry in the model profile. However, this does not affect the intensity of the CDS absorption feature. The comparison to the two-component model and the behavior of $C_{\mathrm{s}}$ show that the role of thermal conduction increases with time, which is the expected result. Interestingly, the result depends rather weakly on $\mu$. This is related to the canceling of effects of the variation of $\mu$ in the thermal excitation of hydrogen and the column density of the third component. We performed modeling of the CDS HV 
absorption adopting the lower boundary velocity, $v_{\mathrm{c}}=11,000 \mathrm{~km} \mathrm{~s}^{-1}$, and found that in this case deceleration of the CDS is too slow, which leads to a larger velocity of the CDS HV absorption on day 102 than is observed. The value $v_{\mathrm{c}}=13,000 \mathrm{~km} \mathrm{~s}^{-1}$ is about the least that provides the required deceleration rate of the CDS.

Although the parameter values used for the third component are reasonable, the modeling cannot be considered as proof that thermal conduction actually dominates the excitation of the CDS material at a late epoch - the model contains many assumptions. Yet the need for an additional source of excitation of hydrogen responsible for the HV absorption is an established result. Thermal conduction is a natural mechanism strongly suggested by the inevitable RT instability and mixing of the CDS matter with hot gas from the forward shock. On the other hand, the additional excitation might at least partially be related to enhanced soft X-ray radiation from hot dense inhomogeneities produced by the RT mixing and heated by thermal conduction up to $\sim 10^{(6-7)} \mathrm{K}$.

\subsection{CDS absorption as a wind probe}

The identification of the features produced by $\mathrm{H} \alpha$ and $\mathrm{H} \beta$ absorption in the CDS provides an efficient tool for the direct determination of the velocity of the $\mathrm{SN} /$ wind interface in SNe IIP. With this value in hand we are able to obtain the wind parameter $w$ using the thin shell model of the deceleration. Of course, the method assumes that the ejecta parameters $M, E$, and $k$ are known.

To facilitate the application of this method we use the self-similar solution. This description is valid at the late photospheric epoch, when the initial conditions related to the structure of the outermost ejecta layers are already 'forgotten.' The self-similar evolution of the CDS radius suggests $R=A t^{\omega}$, with $\omega=(k-3) /(k-2)$ Chevalier 1982a, b; Nadyozhin 1985). The factor $A$ for the adopted density distribution (eq. 1) is

$$
A=\left[\frac{2 M v_{0}^{(k-3)}}{(k-4)(k-3) w C_{m}}\right]^{1 /(k-2)},
$$

were $C_{m}$ is defined by equation (3) and $v_{0}$ by equation (21). The parameters for SN 1999em are as before: $M=19 M_{\odot}, E=1.3 \times 10^{51} \mathrm{erg}$, and $k=9$. The radial velocity of $\mathrm{HV}$ absorption is calculated both for the Lambert photosphere (eq. [7) and with limb darkening described by the first Chandrasekhar approximation. The evolution of the photospheric velocity is set according to Leonard et al. (2002a). The observational estimates of the radial velocity of $\mathrm{HV}$ absorption of $\mathrm{H} \alpha$ are obtained using the spectra reported by Leonard et al. (2002a). The best fit for the wind density parameter is found to be $w=0.99 \pm 0.09$ (Fig. 12). 
This estimate is in a good agreement with the value derived from the ejecta $\mathrm{HV}$ absorption of $\mathrm{H} \alpha(w=1.00 \pm 0.05)$ and the He I $10830 \AA$ line $(w=1.15 \pm 0.07)$. Uncertainties in the limb darkening law and in the photospheric velocity do not affect the derived value of the wind density.

In the case of SN 2004dj we use the same model as before: $M=19 M_{\odot}, E=0.67 \times 10^{51}$ erg, and $k=9$. The radial velocities of $\mathrm{HV}$ absorption are measured from spectra obtained by Vinko et al. (2006). The best fit wind parameter is $w=1.35 \pm 0.05$ (Fig. 12). This agrees with the value $w=1.27 \pm 0.1$ obtained for SN 2004dj from the ejecta $\mathrm{HV}$ absorption of $\mathrm{H} \alpha$.

The coincidence of $w$ values found by diagnostics based on the HV absorption in ejecta and in the CDS is remarkable because the first probe is based on the model of X-ray emission by the reverse shock and the model of the ionization and excitation of ejecta, while the second is based only on the self-similar dynamics of the thin shell.

\section{DISCUSSION AND CONCLUSION}

Our goal was to study signs of CS interaction in the H $\alpha$ and He I $10830 \AA$ absorption lines of SN IIP during the photospheric stage. We developed a model for the ionization and excitation of $\mathrm{H}$ and $\mathrm{He}$ in the unshocked ejecta taking into account time-dependent effects and the irradiation of ejecta by X-rays. The principal result is a prediction that for a typical RSG wind $(w \sim 1)$, the effects of CS interaction in a normal SN IIP are significant and can be detectable at the photospheric stage in both lines. These effects consist of the emergence of: (i) HV absorption in the blue wing of the undisturbed $\mathrm{H} \alpha$ line (absorption 'shoulder') at $t \sim 40-80 \mathrm{~d}$, and (ii) HV absorption in He I $10830 \AA$ at an age $t \sim 20-60$ days at a radial velocity of $\sim 10^{4} \mathrm{~km} \mathrm{~s}^{-1}$. We identify the HV absorption in SN $1999 \mathrm{em}$ in both $\mathrm{H} \alpha$ and He I $10830 \AA$ and derive a wind density parameter $w \approx 1$. HV absorption in $\mathrm{H} \alpha$ is identified in SN 2004dj as well and the value of the wind density parameter is again found to be $w \approx 1$.

The second important finding is that, in addition to the ejecta, absorption in the CDS formed at the SN/wind interface plays an important role in the HV feature, especially at the late photospheric phase. This component of the HV absorption is manifested as a notch in the HV absorption of $\mathrm{H} \alpha$ in SN 1999em and SN 2004dj after about day 60. A similar notch is seen in the $\mathrm{H} \beta$ line of these SNe. We developed a model that produces the notch in both $\mathrm{H} \alpha$ and $\mathrm{H} \beta$ at the different epochs of SN 2004dj which suggests a growing role for thermal conduction in the heating of the mixed CDS gas. The wind densities estimated from the observed velocities of HV absorption produced by the CDS in SN 1999em and SN 2004dj are consistent with the densities derived from the HV absorption produced by unshocked ejecta. 
The identification of $\mathrm{HV}$ absorption in $\mathrm{H} \alpha$ and $\mathrm{H} \beta$ in SN 1999em has been discussed by Leonard et al. (2002a). Here, we identified $\mathrm{HV}$ absorption in $\mathrm{H} \alpha$ and $\mathrm{H} \beta$ in $\mathrm{SN} 2004 \mathrm{dj}$ at a velocity $\sim 3000 \mathrm{~km} \mathrm{~s}^{-1}$ lower than in SN $1999 \mathrm{em}$. The difference provides an additional argument against a possible metal line origin for these features.

Leonard et al. (2002a) claim that a similar component is also seen in the Na I doublet. Indeed, there is a weak dip with a depth of $\sim 0.02$ at the right velocity, $\sim-11,500 \mathrm{~km} \mathrm{~s}^{-1}$, if it is associated with the NaI $5890 \AA$ doublet. We calculated the optical depth of the Na I $5890 \AA$ doublet for SN $1999 \mathrm{em}$ on day 60 in the model of absorption by the CDS and found that the narrow CDS component in this line has an optical depth of $\sim 0.1$, which means that after broadening by about 10 times the depth of absorption should become equal to $\sim 0.01$, comparable to the observed notch. However, we did not find a similar notch in the Na I line of SN 2004dj, or in any other SN IIP. At present, the reality of the HV absorption in the NaI $5890 \AA$ doublet remains uncertain.

We examined other published spectra of SNe IIP at the photospheric epoch and found evidence for HV absorption in the H $\alpha$ blue wing of SN 1985P (Chalabaev \& Cristiani 1987), in both $\mathrm{H} \alpha$ and He I $10830 \AA$ of SN $1995 \mathrm{~V}$ (Fassia et al. 1998), in H $\alpha$ of SN 2003gd, and SN 2006ov (Li et al. 2007). Available spectra of SN 1999gi do not cover the range $50-70$ days when the most pronounced CS interaction effects should be observed. In noisy spectra on day 39 and 89 reported by Leonard et al. (2002b), we do not find unequivocal evidence for $\mathrm{HV}$ absorption in $\mathrm{H} \alpha$. We checked spectra of SN 2004et (Sahu et al. 2006) and did not find convincing evidence for $\mathrm{HV}$ absorption in $\mathrm{H} \alpha$ or $\mathrm{H} \beta$. Early stage $(20-40 \mathrm{~d})$ spectra of SN 2004et show a shallow absorption in the $\mathrm{H} \alpha$ blue wing rapidly evolving towards the red. However the Si II 6347, $6371 \AA$ doublet may be responsible for this absorption. At a later epoch, a weak notch at $6282 \AA$ is present but unfortunately it coincides with the telluric absorption $6281.7 \AA$ line and the identification with HV absorption is doubtful. Sahu et al. (2006) consider the notch in $\mathrm{H} \alpha$ of SN 2004et to be real and remark that it might be related to CS interaction.

If the HV absorption in SN 2004et is absent, the question arises why the dense wind suggested by a relatively high radio luminosity of this SN compared to SN 2004dj and SN 1999em (Chevalier et al. 2006) is not revealed optically. The answer may be that the wind is so dense that the HV absorption is strong and gets merged with the main absorption as in the model case of $w=4$ (Fig. 2). This conjecture is consistent with the strong $\mathrm{H} \beta$ absorption observed in SN 2004et (Sahu et al. 2006). A direct test of the proposed explanation might be the detection of strong HeI $10830 \AA$ absorption in SN 2004et. Unfortunately, to our knowledge, observations of the He I $10830 \AA$ region in SN 2004et are lacking. Interestingly, SN 2006my (Li et al. 2007), which does not show HV absorption, also has strong H $\beta$ like SN 2004et. If 
the same mechanism (dense wind, $w \gg 1$ ) explains the absence of the HV absorption in this case, then SN 2006my should demonstrate high radio and X-ray luminosities for a SN IIP.

We did not find signatures of $\mathrm{HV}$ absorption in $\mathrm{H} \alpha$ observations of the low-luminosity SN 1999br in spectra taken during the first 42 days after the explosion (Pastorello et al. 2004). In the spectrum on day 42 , the $\mathrm{H} \beta$ line is rather weak and the explanation that has been invoked for SN 2004et (strong CS interaction effect) is not applicable in this case. The likely reason for the absence of HV absorption in SN 1999br is a low wind density, $w<1$. This conjecture has an interesting implication. Since the mass loss rate increases with the stellar mass according to the scaling law of Nieuwenhuijsen \& de Jager (1990), we expect that the main-sequence mass of pre-SN 1999br should be lower than that of SN 1999em.

An interesting result, although model dependent, is that at the late photospheric stage of SN 2004dj ( $t \sim 100 \mathrm{~d}$ ) thermal conduction may be the dominant heating mechanism of the mixed CDS gas in the forward shock. In this regard, we recall the $\mathrm{H} \alpha$ problem in SN 1979C: at an age of $\sim 1$ yr the $\mathrm{H} \alpha$ luminosity was $\sim 0.5$ of the total reverse shock luminosity (Chevalier \& Fransson 1985), which is beyond reasonable values of the H $\alpha$ emission efficiency. A natural solution to the problem of the high $\mathrm{H} \alpha$ luminosity in SN 1979C could be thermal conduction in the mixing layer of the forward shock (Chugai 1997). Another possibility, however, is that the mixing process could bring about enhanced X-ray emission from the hot dense mixed component, which may be another source of additional excitation of hydrogen in the CDS. This soft X-ray component might explain the softening of the X-ray emission observed in SN 1999em (Pooley et al. 2002).

Using Chandra X-ray data, Pooley et al. (2002) conclude that the wind density parameter for SN $1999 \mathrm{em}$ is in the range of $w \sim 1-2$ (or 1.5 - 3 for the distance 11.7 Mpc). Our present value $(w \approx 1)$ is within a factor of 1.5 of the lower limit. This should be considered as good agreement for independent estimates. For SN 2004dj the X-ray data are missing. The interpretation of radio data for SN $1999 \mathrm{em}$ and SN 2004dj in the model of wind free-free absorption indicates that the wind density in SN 2004dj is twice as low as in SN 1999em (Chevalier et al. 2006). This is somewhat discrepant with the present results which indicate similar wind densities for these supernovae. The wind density of SN 1999em may be overestimated compared to SN 2004dj from radio data because of the uncertain maximum in the $8.47 \mathrm{GHz}$ light curve or a possible contribution of synchrotron self-absorption in SN 1999em (Chevalier et al. 2006).

The wind density around SN 1999em and SN 2004dj suggests that the amount of the pre-SN material lost at the RSG phase $\left(\sim 10^{6} \mathrm{yr}\right)$ is moderate in these cases. Assuming the wind velocity of a typical RSG, $u=10 \mathrm{~km} \mathrm{~s}^{-1}$, we find with $w=1$ that at the RSG stage the mass lost by the RSG wind is $\sim 1 M_{\odot}$. With the ejecta mass of $19 M_{\odot}$ (Utrobin 2007), 
$1.4 M_{\odot}$ enclosed in the neutron star, and $<1 M_{\odot}$ lost by the main-sequence wind, the initial mass of SN 1999em progenitor turns out to be about $22-23 M_{\odot}$.

The mass loss rate by massive stars is often estimated using the formula of Nieuwenhuijsen \& de Jager (1990) in which $\dot{M}$ is defined through the stellar luminosity $(L)$, mass $(M)$, and radius $(R)$. For the RSG stage, this formula can be modified using the relation between the mass and luminosity $L / L_{\odot}=74\left(M / M_{\odot}\right)^{2.523}$ obtained from evolution calculations of Mevnet \& Maeder (2003) in the range $15-25 M_{\odot}$ for moderate/no rotation. With this relation, the mass loss rate by Nieuwenhuijsen \& de Jager (1990) becomes $\dot{M}=2 \times 10^{-12}\left(M / M_{\odot}\right)^{3.29}\left(R / R_{\odot}\right)^{0.81} M_{\odot}$ $\mathrm{yr}^{-1}$. Since the luminosity at the RSG stage is determined by the mass of the He core which in turn is determined by the initial mass, we should use the initial mass to estimate $\dot{M}$. For SN 1999em with $M=22 M_{\odot}$ and $R=500 R_{\odot}$ (Utrobin 2007) this formula predicts a mass loss rate $\dot{M} \approx 8 \times 10^{-6} M_{\odot} \mathrm{yr}^{-1}$, i.e., eight times larger than our value. A similar result is expected for SN 2004dj. The disparity between our estimate of the pre-SN mass loss rate and the popular scaling law requires an explanation. The disparity cannot be attributed to a lower metallicity, because the metallicities of NGC 1637 (host galaxy for SN 1999em) and NGC 2403 (host galaxy for SN 2004dj) are approximately solar (Pilyugin et al. 2004). The difference can be reduced by a factor of 1.5 if we adopt a higher velocity for the pre-SN wind, $u=15 \mathrm{~km} \mathrm{~s}^{-1}$ instead of $10 \mathrm{~km} \mathrm{~s}^{-1}$. The choice of $15 \mathrm{~km} \mathrm{~s}^{-1}$ is supported by the wind velocity of the massive RSG Betelgeuse, $u=14.3 \mathrm{~km} \mathrm{~s}^{-1}$ (Mauron 1990). This, however, does not completely resolve the problem. We admit the possibility that the hydrodynamical model of the light curve (Utrobin 2007) might significantly overestimate the mass of ejecta for some unknown reason. In that case, to obtain the wind $w \approx 1$ (for $u=15 \mathrm{~km} \mathrm{~s}^{-1}$ ) the initial mass of the presupernova must be $M=13.5 M_{\odot}$ with an ejecta mass of $M \approx 11 M_{\odot}$ instead of $M \approx 19 M_{\odot}$. With the SN energy reduced accordingly, the depth and position of the $\mathrm{HV}$ absorption remain the same (Fig. 2c).

To conclude, we have found three new probes of the wind density in SNe IIP that rely on spectroscopic observations during the photospheric epoch 20-100 d of (1) the ejecta HV absorption in $\mathrm{H} \alpha$; (2) the ejecta HV absorption in He I $10830 \AA$; and (3) the HV absorption (notch) produced by the CDS. The third method is more practical than the other two because it does not require modeling of the absorption lines; it uses velocity measurements and the self-similar model for the velocity fit. Of course, this method, as well as the other two, assumes that we know the ejecta parameters $(M, E, k)$. However, the application of all three methods may be used to constrain ejecta parameters as well. The first two methods are sensitive to winds with a density parameter $w>0.5$. However in $\mathrm{H} \alpha \mathrm{CS}$ interaction effects saturate for large density $w \gtrsim 4$. Therefore, only He I $10830 \AA$ remains a useful diagnostic tool for a high density wind with $w>2$. For the third method the range of accessible wind density is less certain, because a CDS formed by the initial boundary shell could exist 
in principle even in the case of a low density wind, although the hydrogen excitation and shell density depend on the wind density. Further understanding of the relation between the strength of deep HV absorption and the wind density requires 3D hydrodynamical modeling of the shock breakout phase in the presence of a wind and new spectroscopic observations in both $\mathrm{H} \alpha$ and $\mathrm{He} \mathrm{I} 10830 \AA$ lines.

We thank Daniela Korcakova for sending us spectra of SN 2004dj, Jozsef Vinko for informing us about SN 2004dj spectra in the archive SUSPECT, D. K. Sahu for sending us

spectra of SN 2004et, and the referee for helpful comments on the manuscript. This research was supported in part by NSF grant AST-0307366.

\section{REFERENCES}

Blondin, J. M., \& Ellison, D. C. 2001, ApJ, 560, 244

Chalabaev, A. A., \& Cristiani, S. 1987, in ESO Workshop on the SN 1987A, ed. I.J. Danziger, (Garching: ESO), 655

Chevalier, R. A. 1981, Fund. of Cosmic Phys., 7, 1

Chevalier, R. A. 1982a, ApJ, 258, 790

Chevalier, R. A. 1982b, ApJ, 259, 302

Chevalier, R. A. 1998, ApJ, 449, 810

Chevalier, R. A., \& Blondin J. M. 1995, ApJ, 444, 312

Chevalier, R. A., \& Dwarkadas, V. V. 1995, ApJ, 452, L45

Chevalier, R. A., \& Fransson, C. 1994, ApJ, 420, 268

Chevalier, R. A., \& Fransson, C. 1985, in Supernovae as distance indicators, ed. N. Bartel (Berlin: Springer), 123

Chevalier, R. A., Fransson, C., \& Nymark, T. 2006, ApJ, 641, 1029

Chugai, N. N. 1987, SvAL, 13, 282

Chugai, N. N. 1991, in Supernovae, ed. S.E. Woosley (New York: Springer), 286

Chugai, N. N. 1997, ApSS, 252, 225 
Chugai, N. N., \& Chevalier, R. A. 2006, ApJ, 641, 1051

Chugai, N. N., Chevalier, R. A., \& Lundqvist, P. 2004, MNRAS, 355, 627

Cowie, L. L., \& McKee, C. F. 1977, ApJ, 211, 135

Cox, A. N. 2000, Allen's Astrophysical Quantities (New York: AIP Press)

Dessart, L., \& Hillier, D. J. 2005, A\&A, 437, 667

Dessart, L., \& Hillier, J. 2007, in The Multicoloured Landscape of Compact Objects and their Explosive Progenitors, in press (astro-ph/0610136)

Elmhamdi, A., et al. 2003, MNRAS, 338, 939

Falk, S. F., \& Arnett, W. D. 1977, ApJS, 33, 515

Fassia, A., Meikle, W. P. S., Geballe, T. R., Walton, N. A., Pollacco, D. L., Rutten, R. G. M., \& Tinney, C. 1998, MNRAS, 299, 150

Fransson, C. 1984, A\&A, 133, 264

Graham, J. R. 1988, ApJ, 335, L53

Grasberg, E. K., Imshennik, V. S., \& Nadyozhin, D. K. 1971, Ap\&SS, 10, 28

Hamuy, M., et al. 2001, ApJ, 558, 615

Hanuschik, R. W., Thimm, G., \& Dachs, J. 1988, MNRAS, 234, 41P

Heger, A., Fryer, C. L., Woosley, S. E., Langer, N., \& Hartmann, D. H. 2003, ApJ, 591, 288

Hirschi, R., Meynet, G., \& Maeder, A. 2004, A\&A, 425, 649

Jura, M., \& Kleinmann, S. G. 1990, ApJSS, 73, 769

Korcakova, D., Mikulasek, Z., Kawka, A. et al. 2005, Bul. Var. Stars, 5605, 1

Kozma, C., \& Fransson, C. 1992, ApJ, 390, 602

Leonard, D. C., Kanbur, S.M., Ngeow, C.C \& Tanvir, N.R. 2003, ApJ, 594, 247

Leonard, D. C., et al. 2002a, PASP, 114, 35

Leonard, D. C., et al. 2002b, AJ, 124, 2490

Leonard, D. C., \& Filippenko, A. V. 2001, PASP, 113, 920 
Li, W., Wang, X., Van Dyk, S. D., Cuilandre, J. C., Foley, R. J., \& Filippenko, A. V. 2007, ApJ, submitted (astro-ph/0701049)

Matzner, C., \& McKee, C. F. 1999, ApJ, 510, 379

Mauron, N. 1990, A\&A, 227, 141

Meynet, G., \& Maeder, A. 2003, A\&A, 404, 975

Nadyozhin, D. K. 1985, ApSS, 112, 225

Nieuwenhuijsen, H., \& de Jager, C. 1990, A\&A, 231, 134

Nymark, T. K., Fransson, C., \& Kozma, C. 2006, A\&A, 449, 171

Pastorello, A., et al. 2004, MNRAS, 347, 74

Pilyugin, L. S., Vilchez, J. M., \& Contini, T. 2004, A\&A, 425, 849

Pooley, D., et al. 2002, ApJ, 572, 932

Pun, C. S. J., et al. 1995, ApJS, 99, 223

Rakowski, C. E. 2005, AdSpR, 35, 1017

Sahu, D. K., Anupama, G. C., Srividya, S., \& Muneer, S. 2006, MNRAS, 372, 1315

Spitzer, L. 1962, Physics of fully ionized gases (New York: Wiley and Sons)

Sreenivasan, K. R., Ramshankar, R., \& Meneveau, C. 1989, Proc. R. Soc. Lond. A 421, 79

Sutherland, R. S., \& Dopita, M.A. 1993, ApJS, 88, 253

Utrobin, V. P. 2007, A\&A, 461, 233

Utrobin, V. P., \& Chugai, N. N. 2003, From twilight to highlight: The physics of supernovae, ed. W. Hillebrandt \& B. Leibundgut, (Berlin: Springer), 253

Utrobin, V. P., \& Chugai, N. N. 2002, Astron. Lett., 28, 386

Utrobin, V. P., \& Chugai, N. N. 2005, A\&A, 441, 271

van Loon, J. Th., Cioni, M.-R. L., Zijlstra, A. A., \& Loop, C. 2005, A\&A, 438, 273

Vinko, J., et al. 2006, MNRAS, 369, 1780

Xu, Y., McCray, R., Oliva, E., \& Randich, S. 1992, ApJ, 386, 181 
This preprint was prepared with the AAS IATEX macros v5.2. 
Table 1: Parameters for the models of HV absorption

\begin{tabular}{lccc}
\hline Model & $M$ & $E$ & $w$ \\
& $M_{\odot}$ & $10^{51} \mathrm{erg}$ & \\
\hline $\mathrm{rW}$ & 19 & 1.3 & 0.5 \\
$\mathrm{~W}$ & 19 & 1.3 & 1 \\
$\mathrm{dW}$ & 19 & 1.3 & 2 \\
$\mathrm{vW}$ & 19 & 1.3 & 4 \\
$\mathrm{~mW}$ & 10 & 0.9 & 1 \\
$\mathrm{eW}$ & 19 & 0.3 & 1 \\
\hline
\end{tabular}

Table 2: Model parameters for the notch in SN 2004dj

\begin{tabular}{lcccc}
\hline Day & $\beta$ & $\mu$ & $C_{\mathrm{s}}$ & $\tau_{\mathrm{oc}}$ \\
\hline 67 & 0.5 & 0.1 & 8 & 0.15 \\
70 & 0.5 & 0.1 & 8 & 0.15 \\
86 & 0.6 & 0.1 & 13 & 0.35 \\
102 & 0.7 & 0.1 & 30 & 0.4 \\
\hline
\end{tabular}



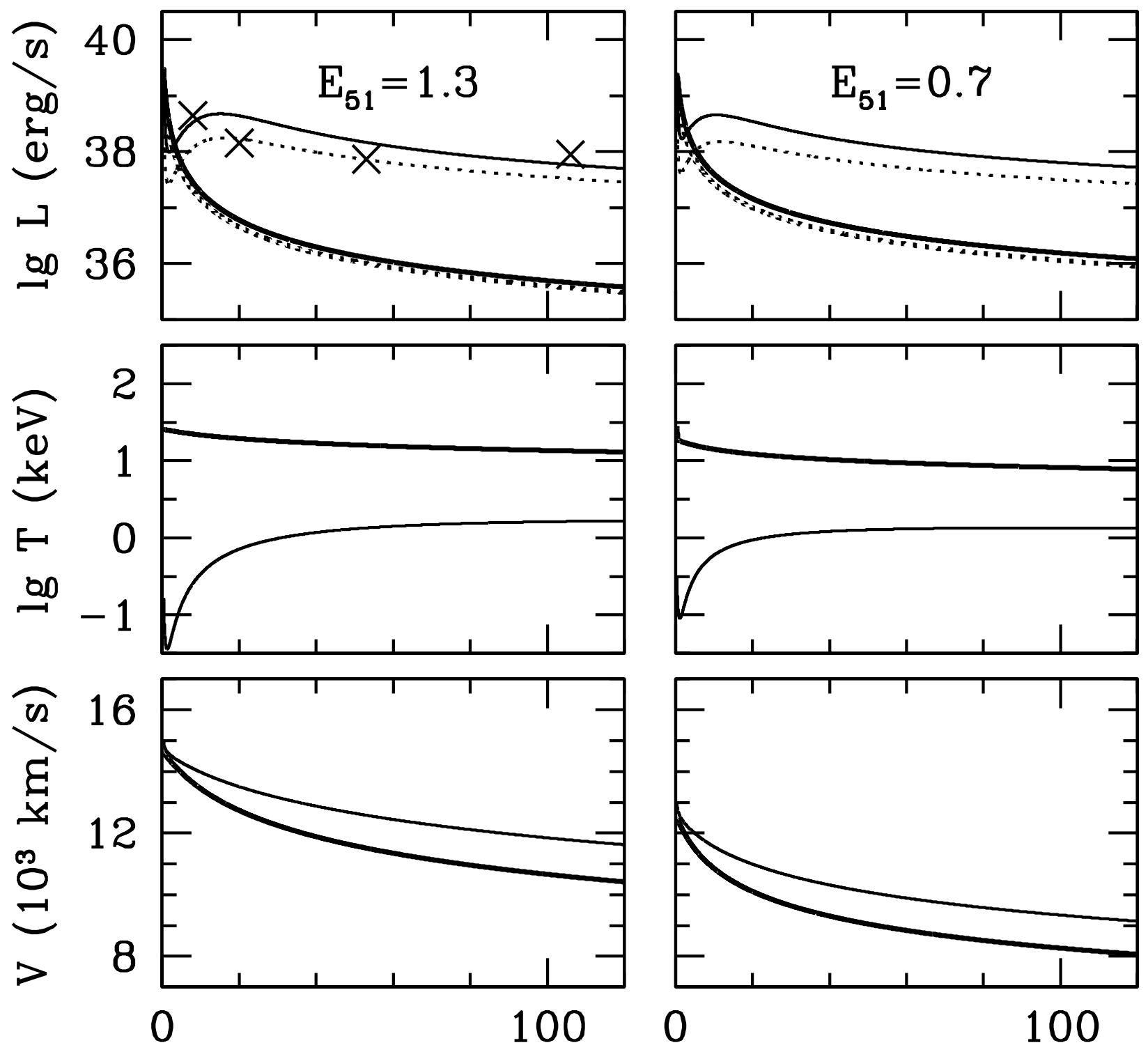

Days

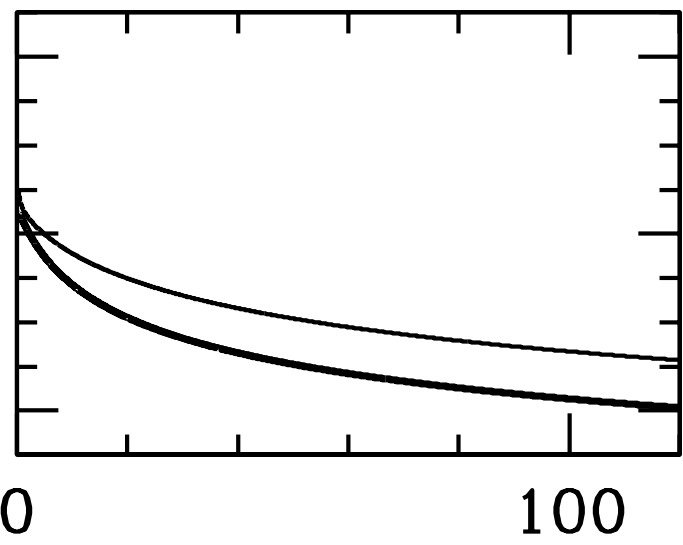

Days

Fig. 1.- Major properties of the interaction model for two values of the kinetic energy of the SN ejecta, $1.3 \times 10^{51} \mathrm{erg}$ (left) and $0.7 \times 10^{51} \mathrm{erg}$. The top panel shows the X-ray luminosity of the forward (thick lines) and reverse (thin) shocks for unabsorbed (solid) and absorbed (dotted) cases. In the left upper panel, Chandra X-ray luminosity measurements of SN 1999em (Pooley et al. 2002) are plotted by crosses. The middle panel displays the electron temperature of the forward (thick lines) and reverse (thin) shocks. The lower panel shows the velocity of the thin shell (thick line) and boundary velocity of the unshocked SN ejecta (thin line). In both cases the X-ray luminosity is dominated by the reverse shock. 


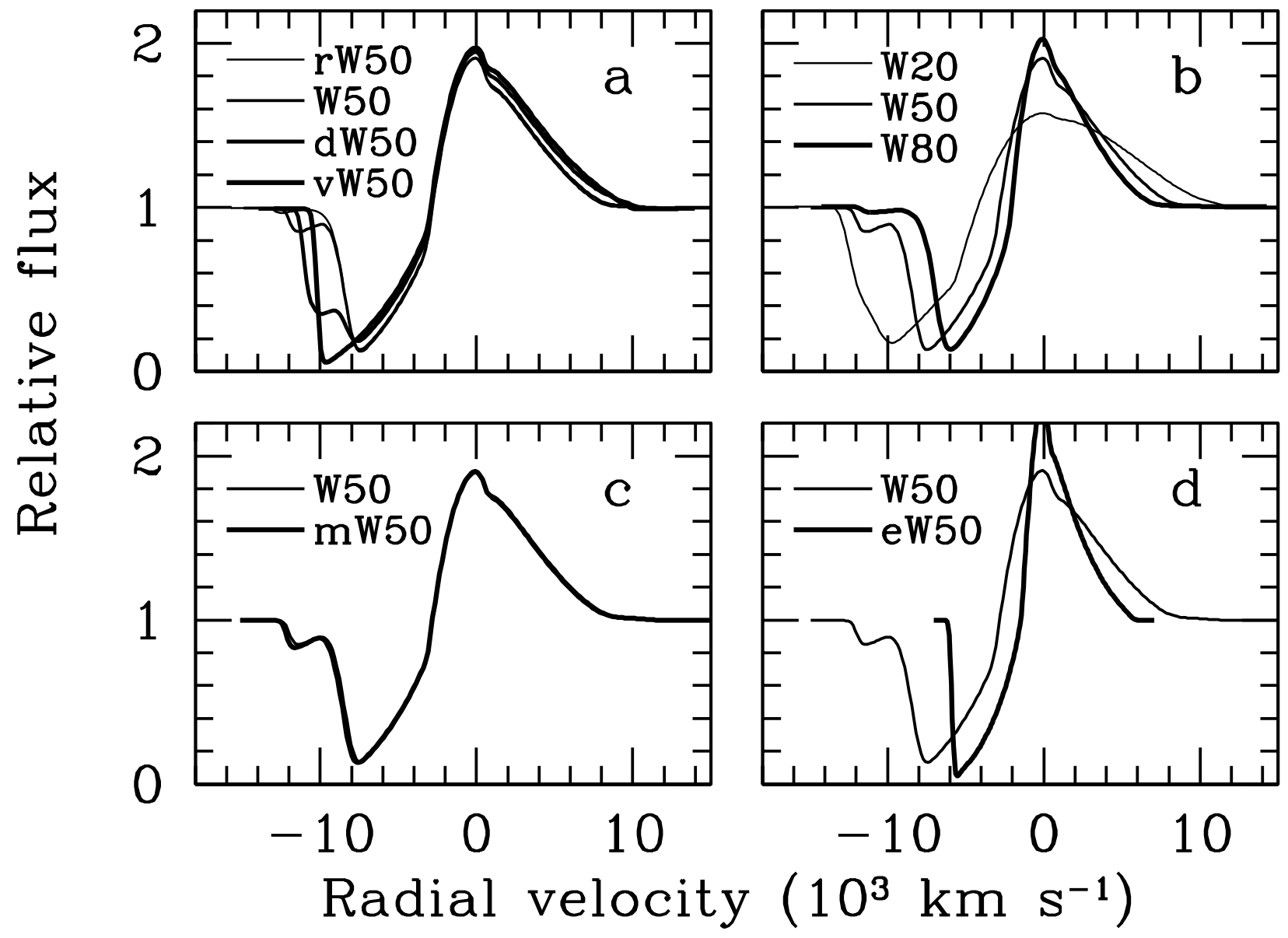

Fig. 2.- The HV absorption in the $\mathrm{H} \alpha$ profile for different models (cf. Table 1). Panel $a$ shows the effect of wind density on day 50; panel $b$ the evolution of the HV absorption in the standard model $\mathrm{W}$ on days 20,50, and 80; panel $c$ shows the negligible effect of low ejecta density for the low mass case mW50; panel $d$ shows the interaction effect in low energy SNe IIP (cf. Table 1). 

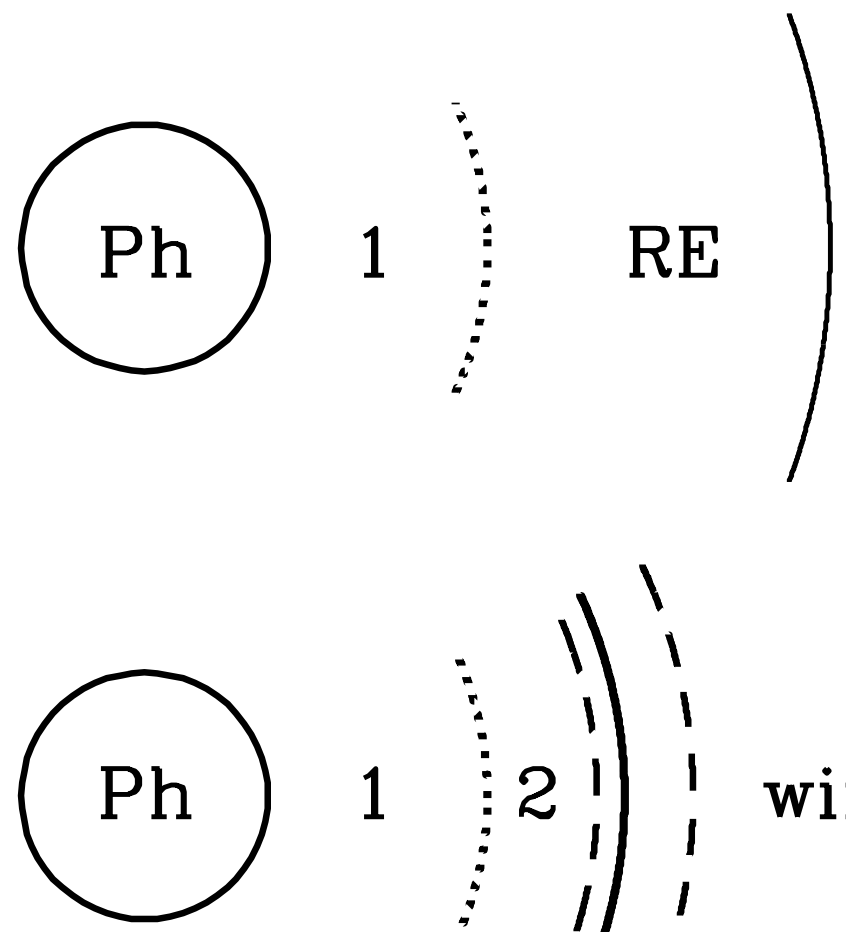
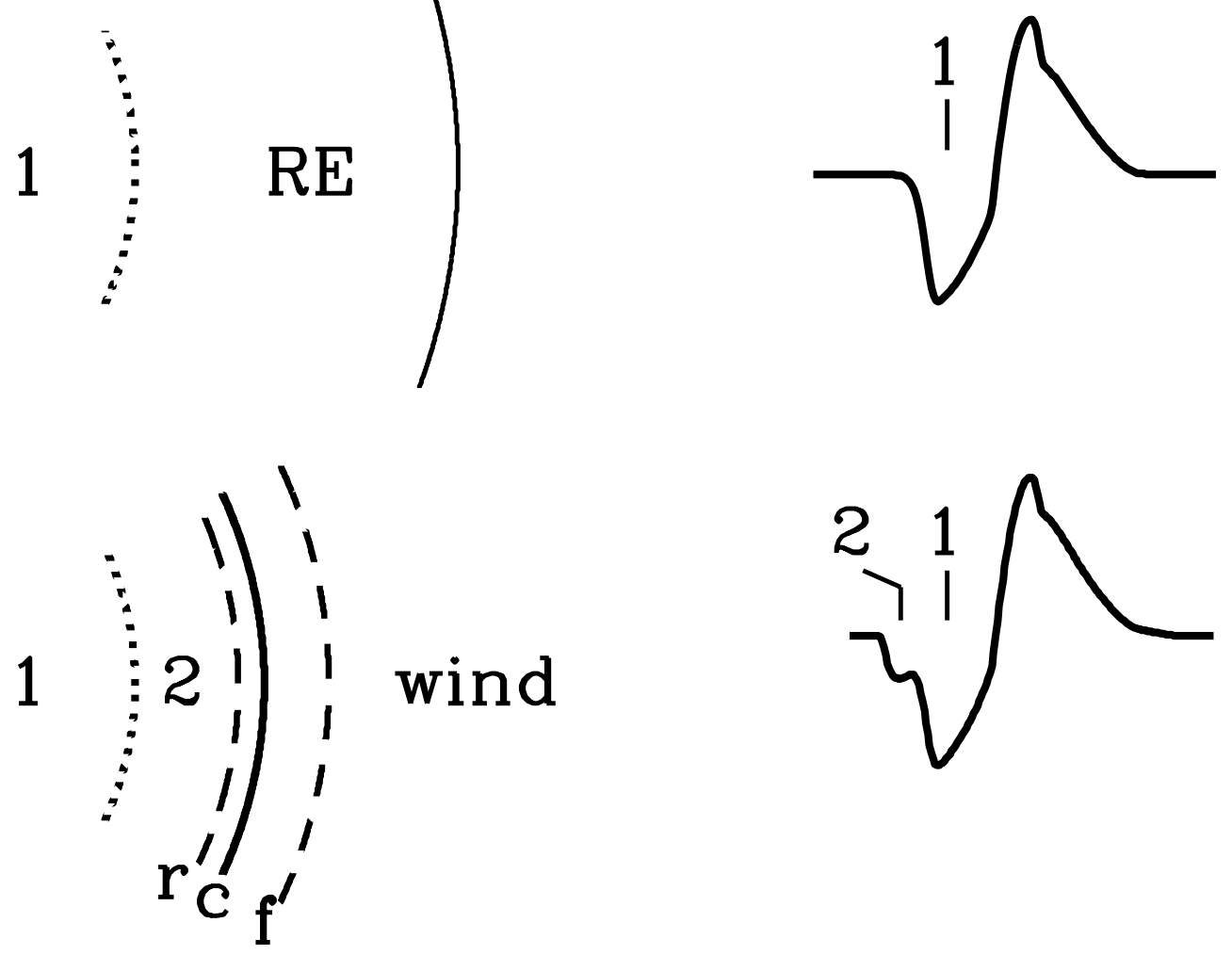

Fig. 3.- Schematic picture of the formation of $\mathrm{H} \alpha$ without and with CS interaction. In the absence of CS interaction (upper), the absorption component forms in the inner layers of ejecta (1) against the photosphere $(P h)$; the outer recombined ejecta $(R E)$ do not contribute to the absorption line profile (upper right). With CS interaction (lower), the double-shocked structure arises at the $\mathrm{SN}$ /wind interface with the forward shock $(f)$, reverse shock $(r)$ and contact surface where the cool dense shell occurs $(c)$. The X-rays, primarily from the reverse shock, ionize and excite the outer layer of ejecta (2) where the HV absorption forms, producing a depression in the blue wing of the undisturbed absorption (bottom right). 


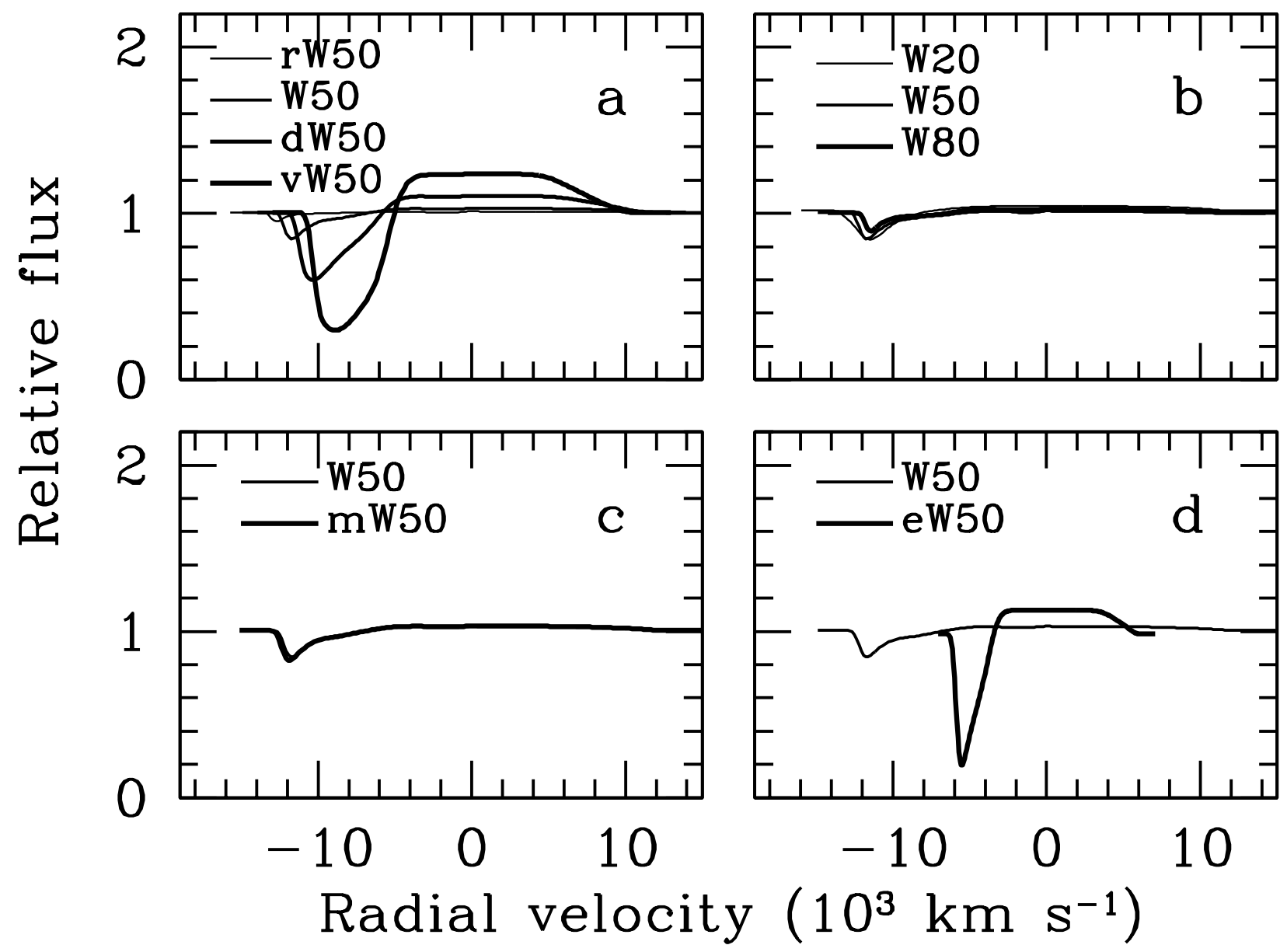

Fig. 4.- The same as Fig. 2 but for the HeI $10830 \AA$ line. 


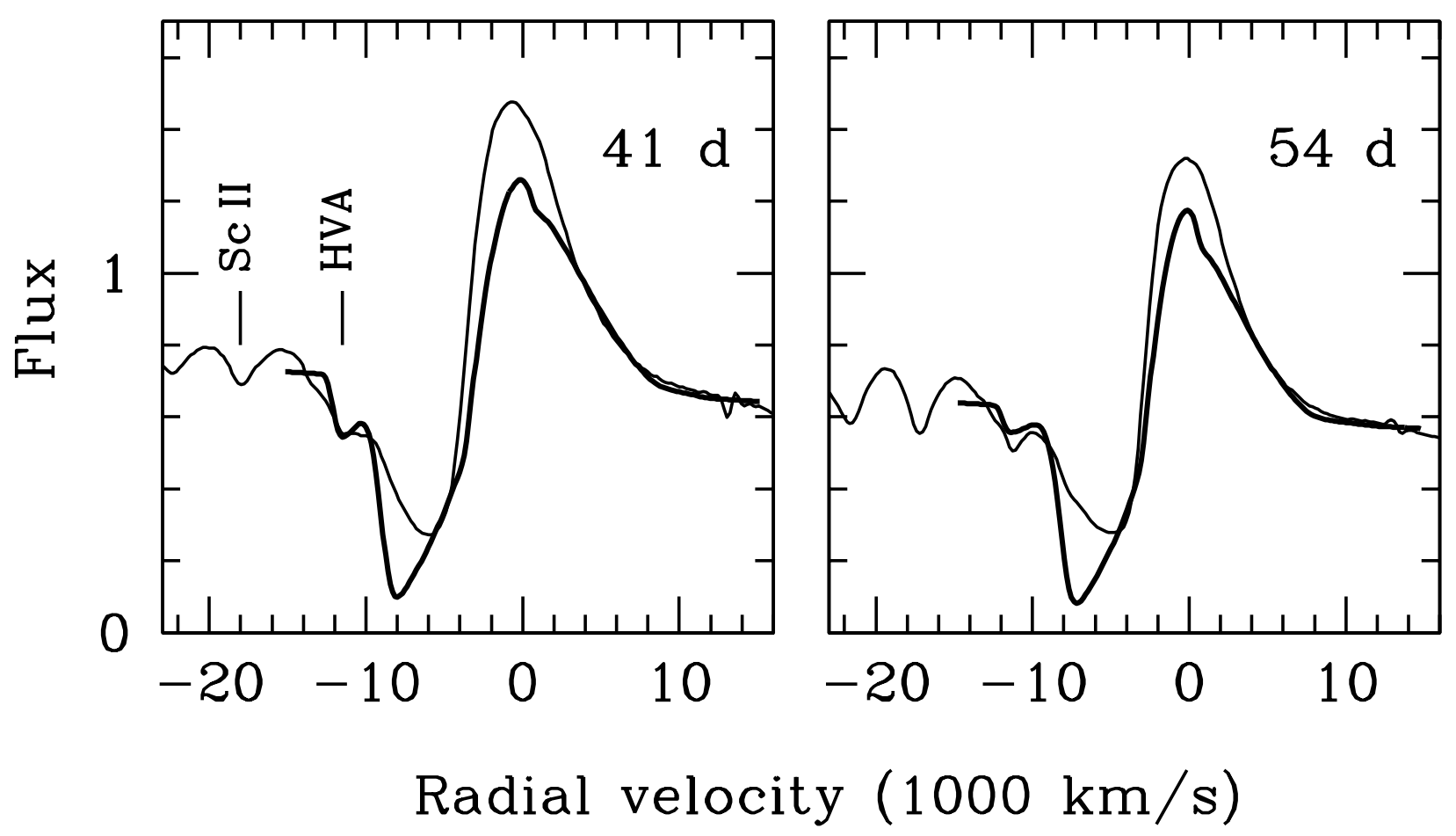

Fig. 5.- Modeling of the $\mathrm{H} \alpha$ profile with HV absorption in SN 1999em on days 41 and 54. The model (thick line) is overplotted on the observations (Elmhamdi et al. 2003). 


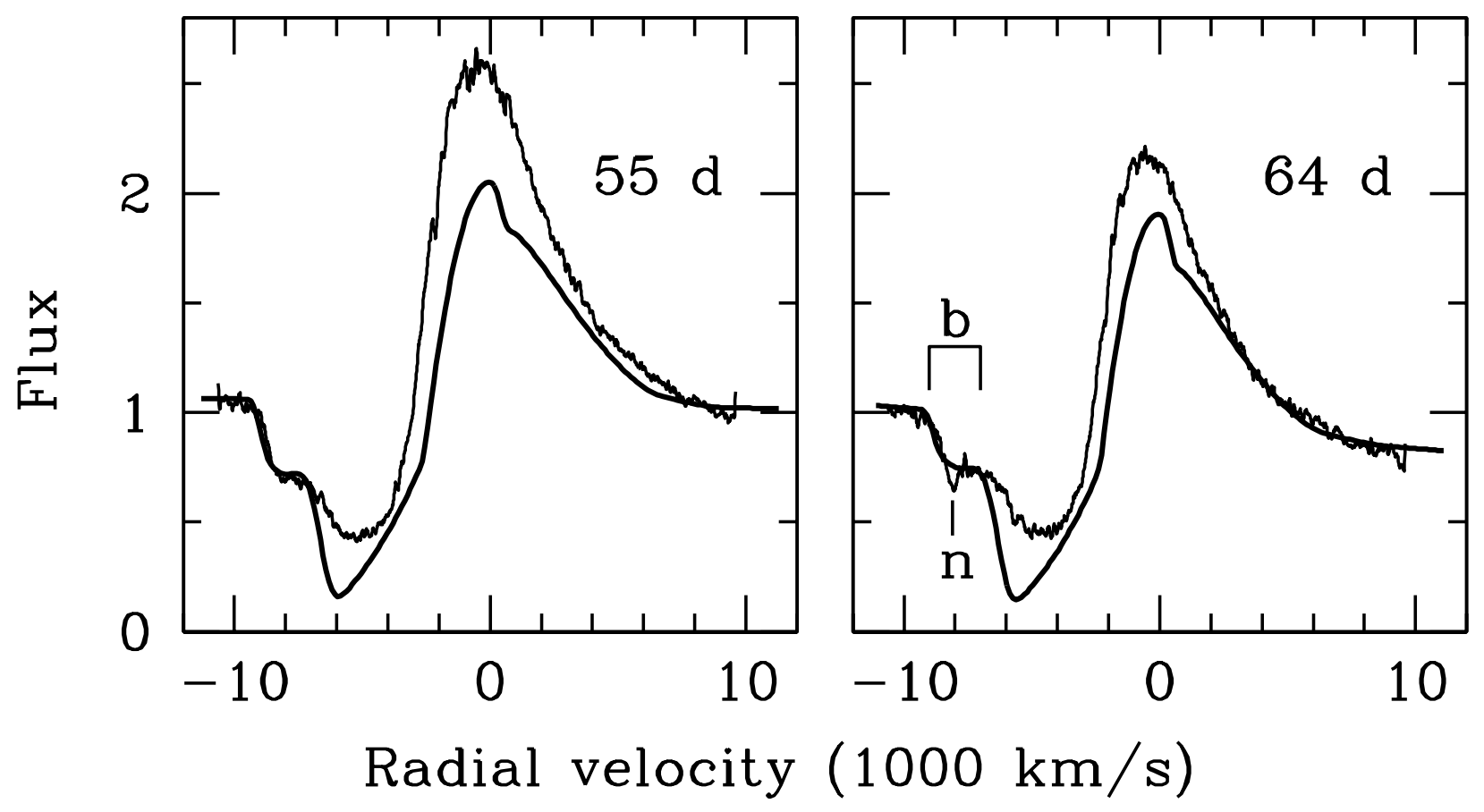

Fig. 6.- Modeling of the $\mathrm{H} \alpha$ profile with HV absorption in SN 2004dj on days 55 and 64. The model (thick line) is overplotted on the observations (Korcakova et al. 2005). On day 64, a notch (n) appears against broader HV absorption (b). 


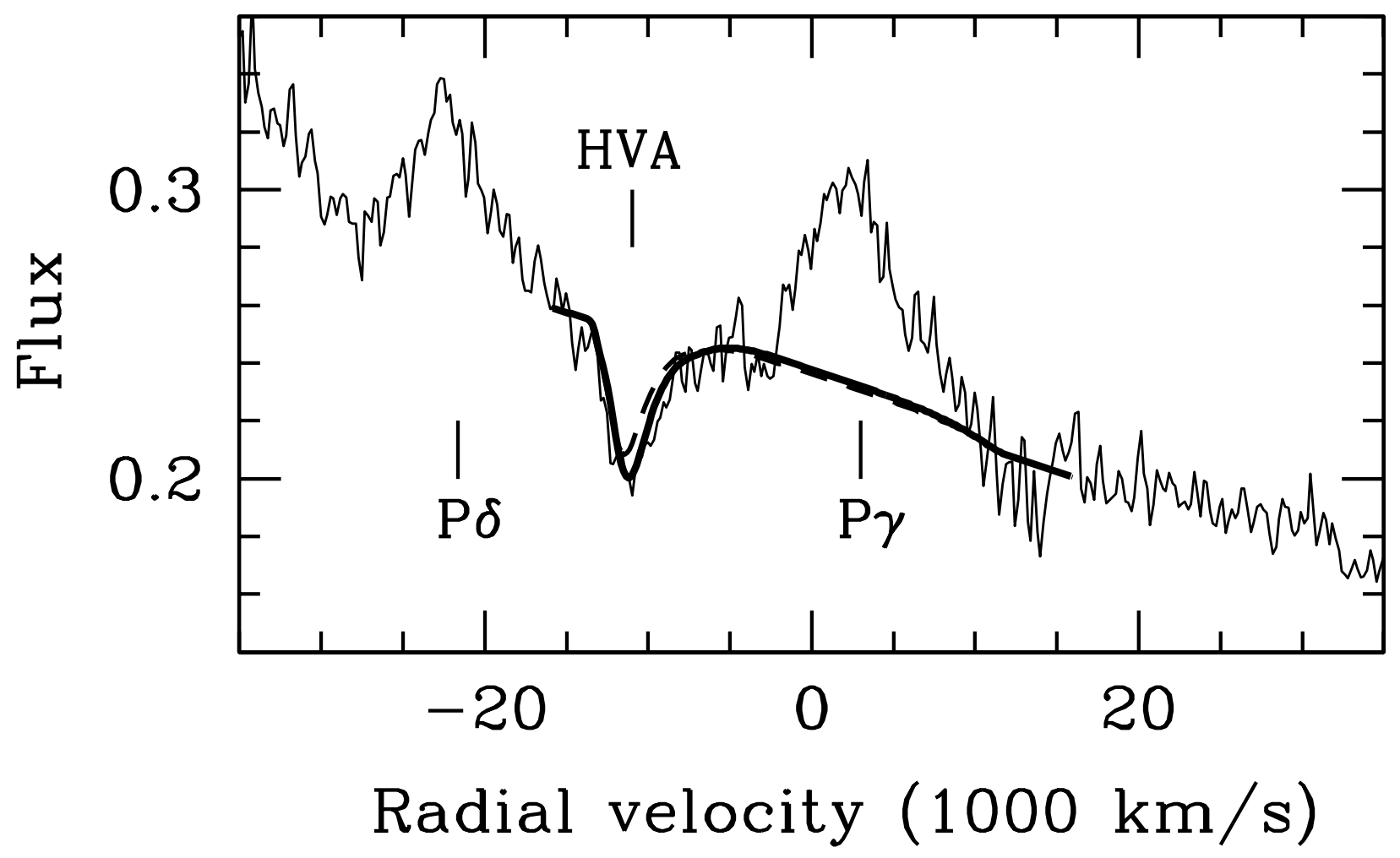

Fig. 7.- High velocity absorption in the HeI $10830 \AA$ line in SN $1999 \mathrm{em}$ on day 20. The model is overplotted on the observations (Elmhamdi et al. 2003). The thick line is the model for $w=1.15$ and the dashed line is the model with $w=1$. Vertical bars show the rest positions of Paschen emission lines. 

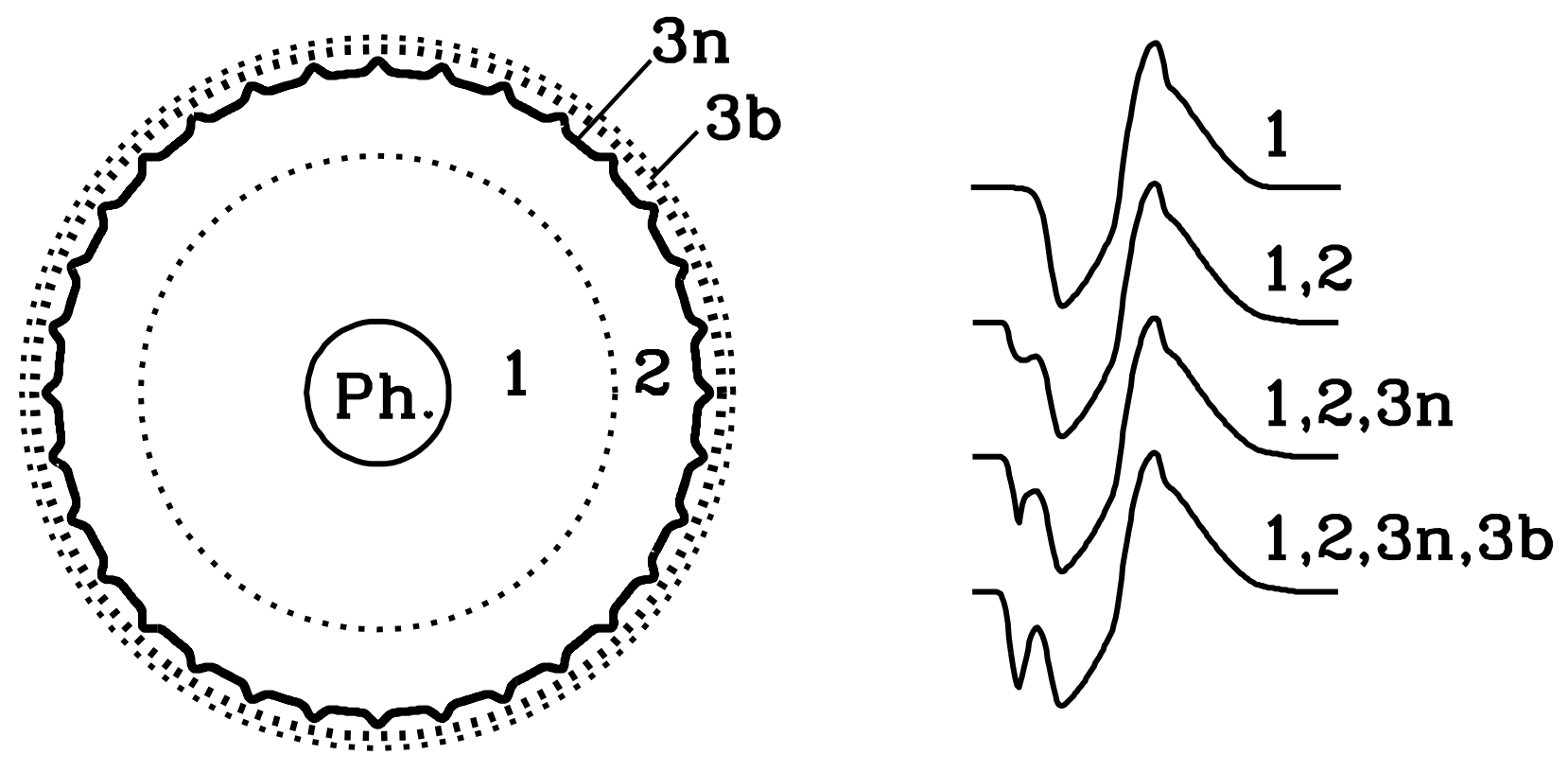

Fig. 8.- Visualization of $\mathrm{H} \alpha$ formation with a contribution from the CDS. Left: The cartoon of a SN depicts the photosphere $(P h$.$) , the undisturbed atmosphere responsible$ for $\mathrm{H} \alpha$ in the absence of CS interaction (1), the layer excited by X-rays from the reverse shock (2) responsible for the shallow ejecta HV absorption, the CDS (3n) responsible for the narrow notch, and the CDS gas mixed into the forward shock region (3b) responsible for the broad notch. Right, upper to lower: The $\mathrm{H} \alpha$ profile without CS interaction (1), with the contribution of the ejecta HV absorption (1,2), with the contribution of a narrow CDS component $(1,2,3 n)$, and with the added broad CDS component $(1,2,3 n, 3 b)$. 


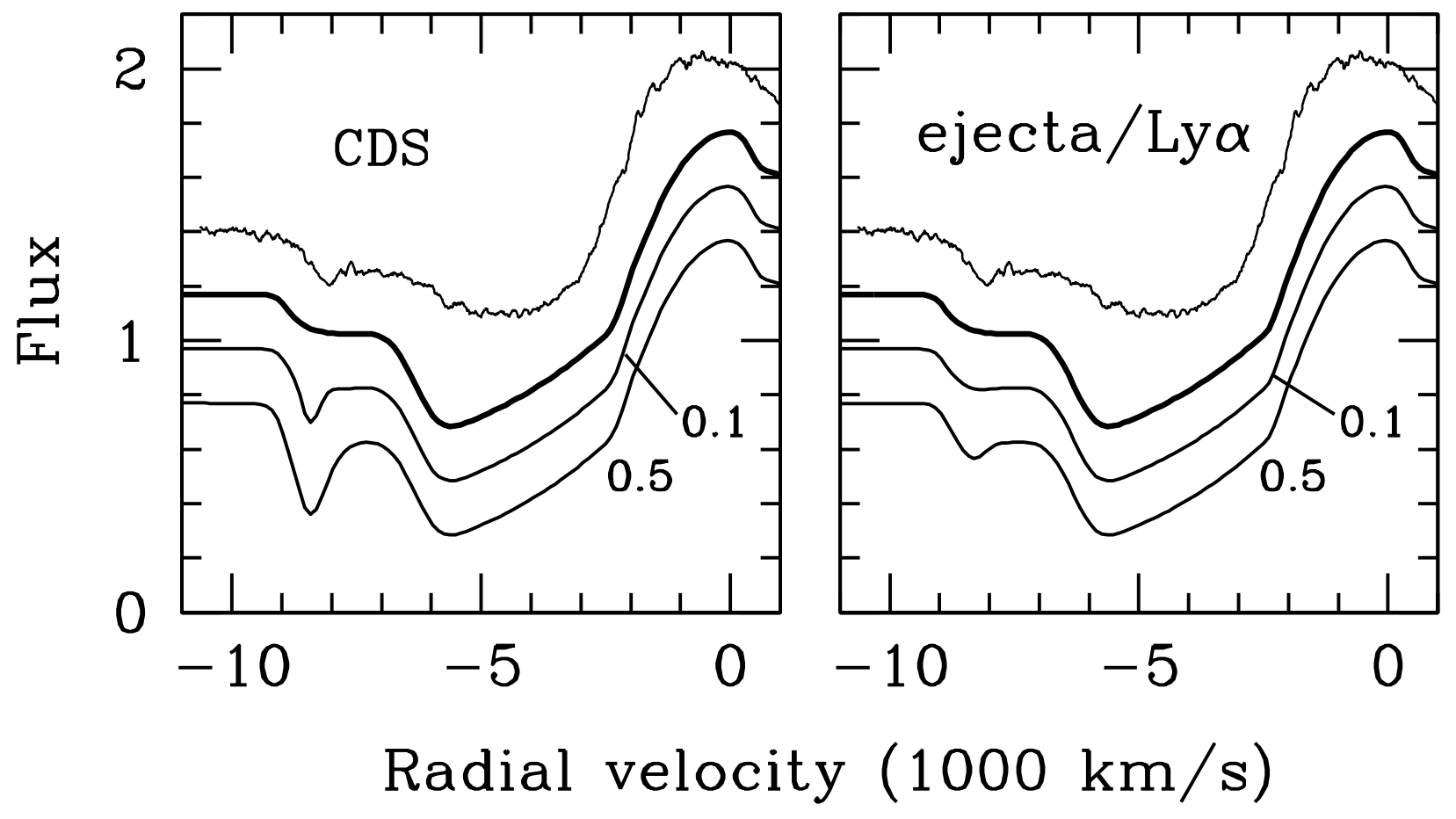

Fig. 9.- Modeling of the HV absorption of $\mathrm{H} \alpha$ in SN 2004dj on day 64. The left panel shows, from top to bottom, the observed spectrum, a model without the CDS absorption, and models with the notch formed in the CDS assuming a two-component CDS with turbulent velocities of 20 and $500 \mathrm{~km} \mathrm{~s}^{-1}$ for different fractions of broad components (0.1 and 0.5). On the right panel, the two top lines are the same as on the left, while the next two are models of the notch formed in the unshocked ejecta by Ly $\alpha$ absorption. The curves are labelled by the corresponding efficiency of Ly $\alpha$ emission. 


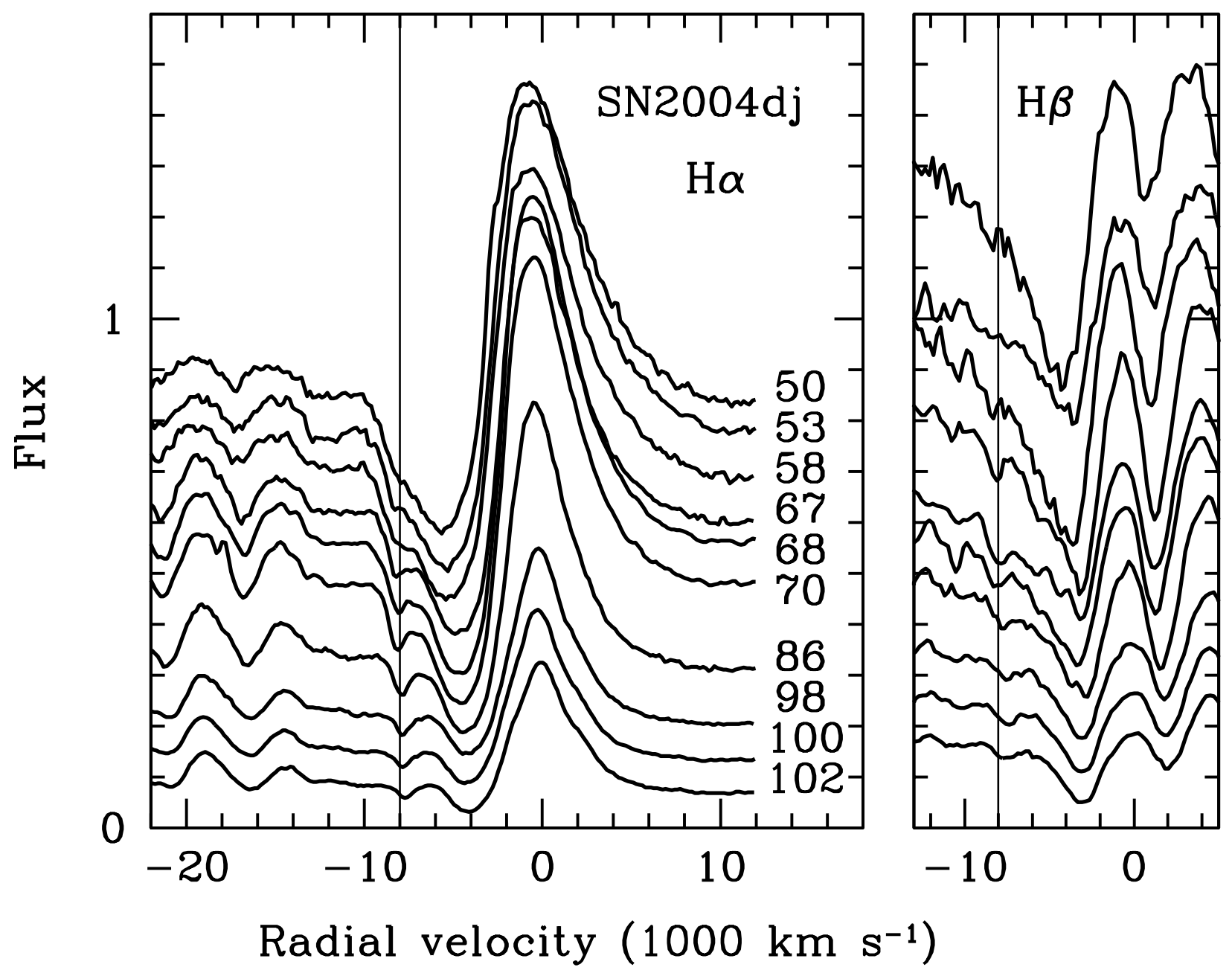

Fig. 10.- A sequence of SN 2004dj spectra (Vinko et al. 2006) in the regions of the $\mathrm{H} \alpha$ and $\mathrm{H} \beta$ lines for the same epochs, showing the HV absorption. Next to the $\mathrm{H} \alpha$ spectra are shown the corresponding ages; the ages of the $\mathrm{H} \beta$ spectra are the same. The notch is seen in both lines at a radial velocity of about $-8000 \mathrm{~km} \mathrm{~s}^{-1}$ starting on day 67 . 


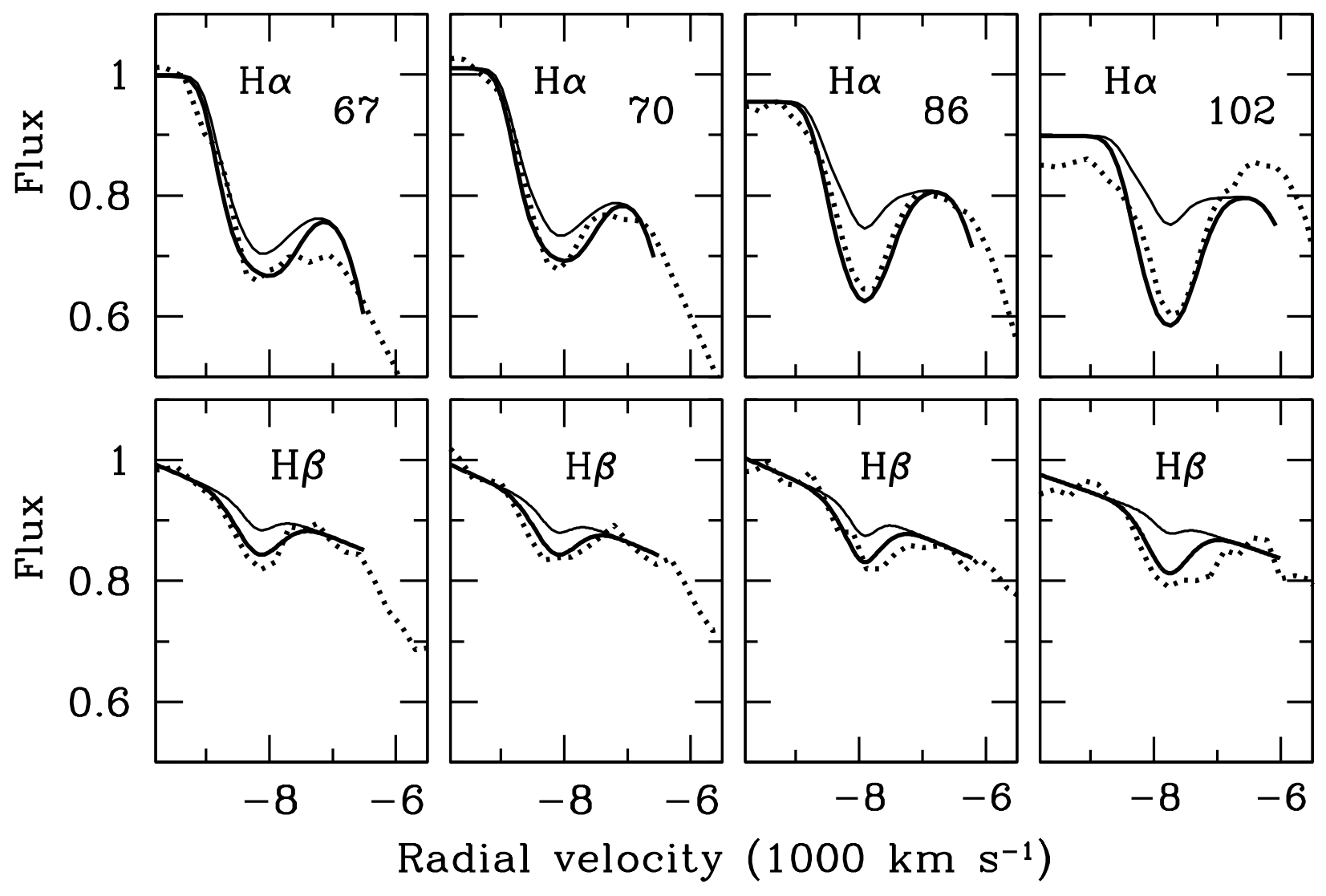

Fig. 11. - Evolution of the HV absorption in the $\mathrm{H} \alpha$ and $\mathrm{H} \beta$ lines of SN 2004dj in a model including absorption by CDS gas. The dotted lines are the observed spectra (Vinko et al. 2006), the thin line is the two-component CDS model composed of narrow and broad CDS components; the thick line is the three-component CDS model (Table 2) with the third component being a broad CDS component powered by thermal conduction. 

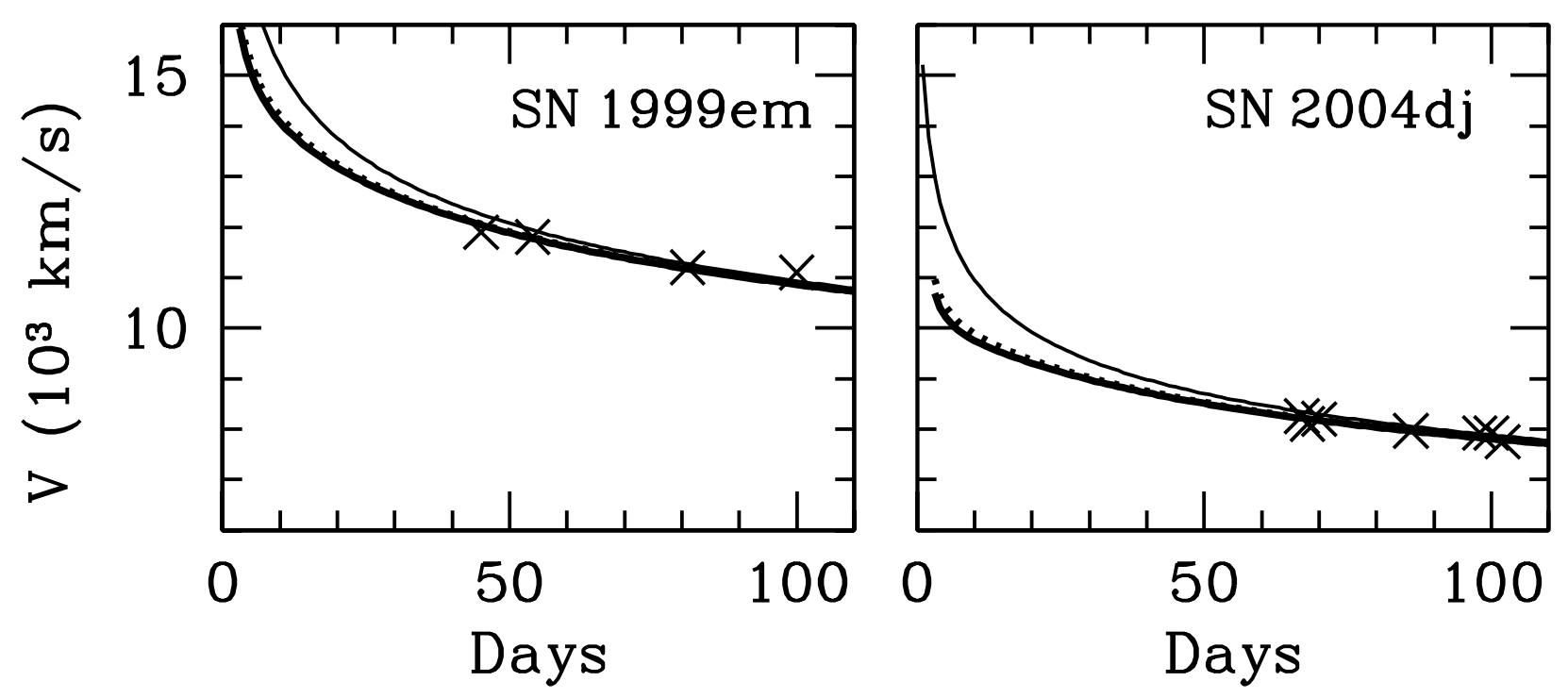

Fig. 12.- Velocity of the HV absorption in H $\alpha$ of SN 1999em and SN 2004dj. The crosses are the velocities of the HV absorption from observed spectra; the thick solid and dotted lines are the velocities of the HV absorption with and without limb darkening in the self-similar model; the thin solid line is the velocity of the CDS in the self-similar model. 